\title{
Density functional theory for a model colloid-polymer mixture: bulk fluid phases
}

\author{
Matthias Schmidt ${ }^{1}$, Hartmut Löwen ${ }^{1}$, Joseph M Brader ${ }^{2,3}$ and \\ Robert Evans ${ }^{2}$ \\ ${ }^{1}$ Institut für Theoretische Physik II, Heinrich-Heine-Universität Düsseldorf, Universitätsstraße 1, \\ D-40225 Düsseldorf, Germany \\ ${ }^{2}$ H H Wills Physics Laboratory, University of Bristol, Royal Fort, Tyndall Avenue, \\ Bristol BS8 1TL, UK
}

Received 10 April 2002, in final form 18 June 2002

Published 27 September 2002

Online at stacks.iop.org/JPhysCM/14/9353

\begin{abstract}
We describe a density functional theory for mixtures of hard sphere (HS) colloids and ideal polymers, the Asakura-Oosawa model. The geometrybased fundamental measures approach which is used to construct the functional ensures the correct behaviour in the limit of low density of both species and in the zero-dimensional limit of a cavity which can contain at most one HS. Dimensional crossover is discussed in detail. Emphasis is placed on the properties of homogeneous (bulk) fluid phases. We show that the present functional yields the same free energy and, therefore, the same fluid-fluid demixing transition as that given by a different approach, namely the freevolume theory. The pair direct correlation functions $c_{i j}^{(2)}(r)$ of the bulk mixture are given analytically. We investigate the partial structure factors $S_{i j}(k)$ and the asymptotic decay, $r \rightarrow \infty$, of the total pair correlation functions $h_{i j}(r)$ obtained from the Ornstein-Zernike route. The locus in the phase diagram of the crossover from monotonic to oscillatory decay of correlations is calculated for several size ratios $q=R_{p} / R_{c}$, where $R_{p}$ is the radius of the polymer sphere and $R_{c}$ that of the colloid. We determine the (mean-field) behaviour of the partial structure factors on approaching the fluid-fluid critical (consolute) point.
\end{abstract}

\section{Introduction}

This paper describes a density functional theory (DFT) for determining the equilibrium properties of the so-called Asakura-Oosawa (AO) model of colloid-polymer mixtures [1, 2]. The model, which was first written down by Vrij [2], treats the colloids as hard spheres (HS) and the globular polymer coils as interpenetrating spheres, as regards their mutual interactions,

${ }^{3}$ Present address: James Franck Institute, University of Chicago, South Ellis Ave. 5640, Chicago, IL 60637, USA. 
but polymers experience an excluded volume (hard) interaction with the colloids. It can be regarded as the simplest, zeroth-order, model of a mixture of colloidal particles and nonadsorbing polymers. Much attention has been paid to the bulk properties of the AO model since this affords an important example of pure entropy-driven fluid-fluid phase separation. When $q$, the size ratio of the radius of the polymer sphere to that of the colloid sphere, is sufficiently large, the mixture separates into colloid-rich (liquid) and colloid-poor (gas) phases. The earliest detailed theoretical treatment of the model was that by Gast et al [3] based on liquid state perturbation theory for the (approximate) one-component version where colloids are assumed to interact via the AO pair potential. An alternative approach was introduced by Lekkerkerker et al [4] based on a free-volume theory for the free energy of the mixture-see section 3.1. These approximate theories, along with some simulation studies of simplified versions of the AO model $[5,6]$, indicate that when the size ratio $q$ is larger than about 0.35 fluid-fluid separation is stable with respect to the fluid-solid transition. Other studies have investigated the equilibrium pair correlation functions in the AO model [5, 7-9] and the formal status of the mapping to a one-component fluid [8]. Although the AO model is highly idealized the variation of bulk phase behaviour with $q$ predicted by studies of the model is in keeping with the experimental results $[10,11]$. More recently attention has shifted towards inhomogeneous mixtures. An effective Hamiltonian for the colloids was obtained by integrating out the degrees of freedom of the polymer with both species subject to external fields $[12,13]$. When the size ratio $q<(2 / \sqrt{3}-1)=0.1547 \ldots$ the effective Hamiltonian contains only one- and two-body (pairwise) contributions and for such mixtures it is straightforward to apply this to problems of adsorption at a hard wall [13]. For larger size ratios many-body terms are present in the effective Hamiltonian for both bulk and inhomogeneous mixtures $[5,8,13]$ and incorporating such terms is rather complicated. Thus treating fluid-fluid interfaces and adsorption phenomena which occur for larger values of $q$ is difficult within the effective Hamiltonian perspective and $a d$ hoc prescriptions have been made [12] in order to calculate the density profile of colloids and the surface tension of the free fluid-fluid interface and compare with experimental data $[14,15]$.

The approach we adopt here for the AO model does not exploit the formal mapping to an effective one-component system of colloids. Rather we treat colloid and polymer on equal footing and develop a DFT specifically tailored for the AO mixture. DFT is recognized as powerful tool for describing the equilibrium properties of inhomogeneous fluids [16, 17]. Most effort has been expended on HS fluids, and the fundamental measure theory (FMT) introduced by Rosenfeld [18] and subsequently refined [19-22] has proved particularly versatile and reliable in a wide variety of applications to both pure HS and to mixtures. The FMT approach has also been applied to parallel hard cubes $[23,24]$. Other recent advances in geometry-based DFT include

(i) penetrable spheres [25], where the interatomic potential is a finite constant when the separation is smaller than the sphere diameter,

(ii) the Widom-Rowlinson model [26], where the unlike species interact via a hard core potential and the like-like interactions are ideal,

(iii) a model proposed by Bolhuis and Frenkel [27] that describes a mixture of HS and infinitely thin needles [28],

(iv) a model of an amphiphilic hard body mixture [29] and

(v) a ternary mixture of colloids, polymers and needles [30].

Our DFT for the AO model is also based on FMT ideas. An earlier letter [31] gave a brief outline of the theory and some results for bulk properties while a second letter described applications to fluid-fluid interfaces and wetting behaviour for the mixture adsorbed at a hard wall [32]. In this paper we provide a comprehensive description of the functional and its bulk properties 
(thermodynamic functions and pair correlation functions) which explains the status of the DFT and which lists explicit expressions for all the relevant quantities required to implement the formalism. Detailed results for interfacial properties obtained from the DFT are given in [9].

Our paper is organized as follows: section 2 is devoted to the theory. We begin in 2.1 with an overview of DFT and describe the strategy for constructing functionals based on the FMT approach. The AO model is specified in 2.2 and in 2.3 we examine two exact limiting cases, namely the low density expansion and the zero-dimensional limit which corresponds to a small cavity that can hold at most one colloidal sphere. The free energy of the latter case constitutes a generating function for the three-dimensional functional and this is described in 2.4 where the functional for the AO model is presented explicitly. Section 2.5 focuses on the properties of the functional in various limiting cases and, in particular, how it accounts for zero-, one- and two-dimensional density distributions. An alternative derivation of the functional is presented in section 2.6. This is based on the original binary HS functional of Rosenfeld [18] and some observations on the diagrammatic expansions of the pair direct correlation functions for HS and for the AO model. We comment on the procedure of integrating out polymer degrees of freedom in the context of DFT and the nature of an effective one-component functional in 2.7. Section 3 is concerned with the predictions of the DFT for the properties of homogeneous (bulk) fluid phases. In 3.1 we focus on thermodynamic functions and show that the bulk free energy of the mixture is precisely the same as that obtained from the free-volume theory of Lekkerkerker et al [4]. Fluid-fluid demixing is described in 3.2 where it is shown that the spinodal in the $\eta_{\mathrm{p}}, \eta_{\mathrm{c}}$ plane can be obtained analytically. $\eta_{\mathrm{p}}, \eta_{\mathrm{c}}$ refer to the packing fractions of polymer and colloid, respectively. Section 3.3 describes the pair direct correlation functions $c_{i j}^{(2)}(r)$ obtained by functional differentiation of our AO functional; these are given analytically and their behaviour with $\eta_{\mathrm{p}}$ and $\eta_{\mathrm{c}}$ is discussed. In 3.4 we focus on the partial structure factors $S_{i j}(k)$ of the AO mixture and their behaviour when the mixture is close to the consolute (critical) point. Since the $S_{i j}(k)$ are given analytically the correlation length of the bulk fluid can be extracted straightforwardly. Section 3.5 is concerned with the nature of the asymptotic decay of pair correlations in the bulk AO mixture. We present results for the so-called FisherWidom (FW) line, which denotes the line in the phase diagram where the ultimate decay of the total pair correlation functions $h_{i j}(r)$ crosses over from monotonic to oscillatory. Section 3.6 discusses the predictions of the present DFT for the effective interaction (depletion potential) between colloidal particles, contrasting the Ornstein-Zernike (OZ) route with the alternative test-particle route. We conclude in section 4 with a summary and a discussion of some of the advantages and some shortcomings of our approach.

\section{Theory}

\subsection{Overview and strategy}

Within DFT the basic variables that describe the microscopic degrees of freedom of a manybody system are the one-body densities $\rho_{i}(\boldsymbol{r})$ of each species $i: \rho_{i}(\boldsymbol{r}) \mathrm{d} V$ is the average number of particles (of species $i$ ) in an infinitesimal volume element $\mathrm{d} V$ located at position $r$. Clearly $\rho_{i}(\boldsymbol{r})$ can resolve inhomogeneities (spatial deviations from a uniform value) on small length scales. Such inhomogeneous structuring is present in liquids under external influence and is manifestly present in crystalline solids. Introducing the particle positions $\boldsymbol{r}_{i}^{(j)}$, where $j=1, \ldots, N_{i}$, and $N_{i}$ is the total number of particles of species $i$, one defines

$$
\rho_{i}(\boldsymbol{r})=\left\langle\sum_{j=1}^{N_{i}} \delta\left(\boldsymbol{r}-\boldsymbol{r}_{i}^{(j)}\right)\right\rangle,
$$


where the angular brackets denote an appropriate ensemble average over many configurations. Evidently the $\rho_{i}(\boldsymbol{r})$ for a given statepoint (e.g. temperature and chemical potentials $\mu_{i}$ ) contain considerable knowledge about the system. More significant is the fact that the thermodynamic potential of the system is determined solely by the one-body densities. The existence of a functional that converts the functions $\rho_{i}(r)$ to the grand potential $\Omega$ (which is a number) is one of the building blocks of DFT. Within this description, the $\rho_{i}(\boldsymbol{r})$ are basically the system's only degrees of freedom. At first glance this may seem to be a limitation: how can the important structural information contained in higher-body correlations such as the pair distribution functions be obtained, if the theory operates on the one-body level? The answer lies in the second building block of DFT: the variational principle states that upon varying the $\rho_{i}(\boldsymbol{r})$, the grand potential functional is a minimum at the true equilibrium densities and its value at the minimum is the true grand potential $\Omega$. The grand potential functional has the form

$$
\Omega\left[\rho_{i}(\boldsymbol{r})\right]=F_{\mathrm{exc}}\left[\left\{\rho_{i}(\boldsymbol{r})\right\}\right]+\sum_{i} F_{\mathrm{id}}\left[\rho_{i}(\boldsymbol{r})\right]+\int \mathrm{d}^{3} r \rho_{i}(\boldsymbol{r})\left[V_{i, \mathrm{ext}}(\boldsymbol{r})-\mu_{i}\right],
$$

where $F_{\text {id }}\left[\rho_{i}(\boldsymbol{r})\right]=k_{\mathrm{B}} T \int \mathrm{d}^{3} r \rho_{i}(\boldsymbol{r})\left[\ln \left(\Lambda_{i}^{3} \rho_{i}(\boldsymbol{r})\right)-1\right]$ is the (Helmholtz) free energy of the ideal gas, $k_{\mathrm{B}}$ is Boltzmann's constant, $T$ is temperature, $\Lambda_{i}$ is the thermal wavelength of species $i$ and $V_{i \text {,ext }}$ is an external potential acting on species $i . F_{\text {exc }}$ is the excess Helmholtz free energy arising from interactions between the particles. As this formalism is an exact reformulation of equilibrium statistical mechanics, the hierarchy of higher-body direct correlation functions can be obtained by functional differentiation of $F_{\text {exc }}$ with respect to the density fields and the equilibrium distribution functions from the $\mathrm{OZ}$ equation. The main benefit of DFT is that powerful approximate theories can be obtained, provided the (generally unknown) excess part $F_{\text {exc }}$ is prescribed. The effects caused by different classes of (time-independent) external potentials can all be treated within the same theory, as $F_{\text {exc }}$ is independent of $V_{i \text {,ext }}$; it is a unique functional of $\left\{\rho_{i}(\boldsymbol{r})\right\}$ for a given choice of interparticle potential functions [16, 17]. Of course, the difficult part of any approximate DFT is finding a suitable prescription or recipe for $F_{\text {exc }}$ that will incorporate the essential physics of short-range correlations (arising from the packing of the particles) and of attractive forces (if these are present) between the particles. However, in all cases, $F_{\text {exc }}$ contains non-local contributions: when varying the density at a given point in space, the system is affected on the length scale of the inter-particle potentials (and beyond that through mediated correlations).

Our present DFT recipe to convert colloid and polymer density profiles to an (approximate) excess free energy for the AO model has the following key features. The non-local character is taken care of by convoluting the actual density fields $\rho_{i}(\boldsymbol{r})$ with various weight functions. The results of this procedure are weighted densities, and only these are subsequently used to obtain the excess free-energy functional, not the bare density fields. This type of weighteddensity approximation (WDA) is now the most common tool to deal with very pronounced inhomogeneities: bare density profiles may change by orders of magnitude over distances as small as a particle diameter [33] and convolutions are a suitable means of treating this behaviour as they exhibit the property of smoothing out. Extensitivity of the free energy is incorporated by expressing $F_{\text {exc }}$ as a spatial integral over an excess free-energy density $\Phi$. This ensures that, over length scales which are large compared with the system's correlation lengths, distinct regions of space give additive contributions to the free energy. In order to connect the free-energy density with the weighted densities, our crucial approximation is to regard $\Phi$ as a simple function (not a functional) of the weighted densities. This feature of the theory is identical to that of Rosenfeld's HS functional [18], and to various recent DFTs mentioned in the introduction. The three main differences between this approach and most other weighted-density $[16,17]$ DFTs are the following. 
(i) Rather than a single weight function, we use a set of weight functions $w_{v}^{i}$ (labelled by $v$ ) for each species $i$. Consequently, we have a set of weighted densities $n_{v}^{i}$ for each species $i$.

(ii) The range of the weight functions (defined as the distance beyond which a weight function vanishes) is determined not by the range of interactions, but by the radii of the particles.

(iii) The free-energy density $\Phi$ is not modelled as a product of a bare density times a free energy per particle, but as a function only of the weighted densities $n_{v}^{i}$.

Our DFT is constructed specifically to describe the AO model, i.e. the forms of the $w_{v}^{i}$ and of $\Phi$ are specific to this model. These are obtained by imposing the correct behaviour of the functional in several different cases, where the exact behaviour of the AO model is known. The low density (virial) expansion enables us to obtain the explicit form of the weight functions. As we shall see below, these are the same as for HS [18]. The other case is a situation of extreme confinement, where the particles of the system are only allowed to access a single location in space. This zero-dimensional limit is reminiscent of confining a particle to a crystal lattice site and corresponds to imposing the density distribution for a particle in a small cavity whose dimensions are of the same size as the particle. For HS the importance of this limit was demonstrated by Rosenfeld et al $[19,20]$. Later it was shown that the original Rosenfeld functional [18], as well as improved versions, can be obtained from a systematic treatment of superpositions of (up to three) such density peaks [21, 22].

\subsection{Specification of the Asakura-Oosawa model}

The AO model describes colloids as hard impenetrable spheres and polymers as effective particles with spherical shape. These polymeric spheres are ideal (non-interacting) amongst themselves, but experience a hard core repulsion with the colloids. Clearly, the model is idealized, but it captures the essential physics of real colloid-polymer mixtures. In particular, the AO model describes polymer coils at the theta point, where repulsion is balanced by attraction, such that the effective interaction between polymers (as expressed by their second virial coefficient) vanishes. The AO model should also be regarded as a useful zeroth-order reference system for a wide variety of complex fluids where soft penetrable particles move in the space between hard bodies.

Thus, we consider a mixture of $N_{\mathrm{c}}$ colloids with radii $R_{\mathrm{c}}$, and $N_{\mathrm{p}}$ polymers, with radii $R_{\mathrm{p}}$, interacting via pair potentials $V_{i j}$, with $i, j=\mathrm{c}, \mathrm{p}$, contained inside a (large) volume $V$. The Hamiltonian consists of (trivial) kinetic energy terms and a sum of interaction terms:

$H\left(\left\{\boldsymbol{R}_{i}, \boldsymbol{r}_{j}\right\}\right)=\sum_{i<j}^{N_{\mathrm{c}}} V_{\mathrm{cc}}\left(\left|\boldsymbol{R}_{i}-\boldsymbol{R}_{j}\right|\right)+\sum_{i}^{N_{\mathrm{c}}} \sum_{j}^{N_{\mathrm{p}}} V_{\mathrm{cp}}\left(\left|\boldsymbol{R}_{i}-\boldsymbol{r}_{j}\right|\right)+\sum_{i<j}^{N_{\mathrm{p}}} V_{\mathrm{pp}}\left(\left|\boldsymbol{r}_{i}-\boldsymbol{r}_{j}\right|\right)$,

where $\left\{\boldsymbol{R}_{i}\right\}=\left\{\boldsymbol{r}_{i}^{(\mathrm{c})}\right\}$ denotes colloid and $\left\{\boldsymbol{r}_{i}\right\}=\left\{\boldsymbol{r}_{i}^{(\mathrm{p})}\right\}$ polymer coordinates.

The interaction potential between colloids is hard: $V_{\mathrm{cc}}(r)=\infty$ if $r \leqslant 2 R_{\mathrm{c}}$, and zero otherwise. The interaction between colloids and polymers is also hard: $V_{\mathrm{cp}}(r)=\infty$, if $r \leqslant R_{\mathrm{c}}+R_{\mathrm{p}}$, and zero otherwise. The interaction between polymers vanishes for all distances [2]: $V_{\mathrm{pp}}(r)=0$. Since all the interactions between particles are either hard or ideal, temperature $T$ plays no role in determining phase behaviour or structure and the thermodynamic state of the bulk system is governed by the packing fractions of colloids, $\eta_{\mathrm{c}}=4 \pi N_{\mathrm{c}} R_{\mathrm{c}}^{3} /(3 \mathrm{~V})$, and of polymers, $\eta_{\mathrm{p}}=4 \pi N_{\mathrm{p}} R_{\mathrm{p}}^{3} /(3 V)$. The properties of the system are governed by the size ratio $q=R_{\mathrm{p}} / R_{\mathrm{c}}$, which is the only adjustable parameter in the model. Unlike other theoretical treatments which are based on some integrating out of polymer degrees of freedom [3-5, 12, 13] the present DFT of the AO model treats colloid and polymer on equal footing. 


\subsection{Two exact limiting cases}

2.3.1. Low density expansion. For small densities of all species, $\rho_{i} \rightarrow 0$, the probability that particles interact is small and for uniform fluids this enables one to make the systematic virial expansion of thermodynamic functions in powers of the (bulk) densities. The lowest order term governs the low density behaviour and for the excess free energy this is simply $\beta F_{\text {exc }} / V=-\sum_{i j} \rho_{i} \rho_{j} \int \mathrm{d}^{3} r f_{i j}(\boldsymbol{r}) / 2$, where $f_{i j}(r)=\exp \left(-\beta V_{i j}(r)\right)-1$ are the Mayer functions, with $\beta=1 / k_{\mathrm{B}} T$, and the summations run over all species. A similar expansion can be performed for inhomogeneous systems [16-18]. For a mixture the lowest-order term is

$$
\beta F_{\text {exc }}\left[\left\{\rho_{i}(\boldsymbol{r})\right\}\right]=-\frac{1}{2} \sum_{i j} \int \mathrm{d}^{3} r \mathrm{~d}^{3} r^{\prime} \rho_{i}(\boldsymbol{r}) f_{i j}\left(\left|\boldsymbol{r}-\boldsymbol{r}^{\prime}\right|\right) \rho_{j}\left(\boldsymbol{r}^{\prime}\right) .
$$

Note that as the AO model exhibits only hard core interactions, the $f_{i j}$ take on the values -1 and 0 only: $f_{\mathrm{cc}}, f_{\mathrm{cp}}=-1$ if the particle pair overlaps, and are zero otherwise. $f_{\mathrm{pp}}=0$ for all separations $r$. Equation (4) sums double convolutions of the density profiles, where the Mayer function is the convolution kernel. Although the functional is only second order in densities, an important feature is already present: the range of non-locality is the range of interactions.

2.3.2. Zero-dimensional limit. In statistical physics it is a common simplification to consider models in reduced spatial dimensionality $d$. Often two-dimensional systems are simpler to tackle than three-dimensional systems, and one-dimensional systems are usually simpler than two-dimensional systems. Reduced dimensionalities not only simplify theoretical treatments, but are also realized in nature, e.g. in films (two dimensions) or inside channels (one dimension) or cavities (zero dimensions). A versatile DFT should be able to describe accurately all situations of reduced dimensionality. In order to model the ultimate dimensional crossover to zero dimensions a special limit was introduced $[19,20]$. In this so-called zero-dimensional limit the system is confined in all three spatial dimensions, such that only a single point in three-dimensional space is accessible for the particles. Physically, the zero-dimensional limit may be realized by a small cavity with rigid walls, that is of particle size. The zerodimensional limit is also similar to that of a particle at a crystal lattice site, where each particle is confined within the cage of its nearest neighbours. Any theoretical description for structure and thermodynamics in highly inhomogeneous three-dimensional situations should be able to reproduce dimensional crossover, even to the extreme limit of a zero-dimensional situation. Clearly, this is a demanding requirement. It has been found, in the case of HS, that one can start with a point and by considering zero-dimensional cavities of increasingly complex shape build up a theory for higher dimensions. A strong guide for the general structure of the functional is provided by Percus's exact one-dimensional functional for hard rods [34]. In the case of HS knowledge of the exact zero-dimensional free energy was sufficient to derive a working theory [21], and was subsequently exploited to derive improved versions [22]. The same methodology was used to tackle other systems including penetrable spheres, which interact with a constant pair potential if they overlap [25], the Widom-Rowlinson model [26] and a needle-sphere mixture [28], as well as models for an amphiphilic [29] and a ternary mixture [30]. Here we follow the same route for the AO model.

To be explicit, we introduce an external potential $V_{\text {ext }}(\boldsymbol{r})=0$ if $|\boldsymbol{r}|<\epsilon$, and $\infty$ otherwise. Each particle's centre is then allowed to move inside a sphere of volume $4 \pi \epsilon^{3} / 3$. We consider the limit $\epsilon \rightarrow 0$, so that any particles present in the cavity overlap. In order to derive the zero-dimensional Helmholtz (excess) free energy, we consider the grand partition sum $\Xi$. The only states that are allowed are the following:

(i) the empty state without any particle, 
(ii) the state with exactly one colloidal particle and

(iii) all states without colloids but with an arbitrary number of polymers.

Hence, in that order,

$$
\Xi=1+z_{\mathrm{c}}+\left[\exp \left(z_{\mathrm{p}}\right)-1\right]=z_{\mathrm{c}}+\exp \left(z_{\mathrm{p}}\right)
$$

where the (dimensionless) fugacities are defined here as $z_{i}=\exp \left(\beta \mu_{i}\right) \Lambda_{i}^{-3} 4 \pi \epsilon^{3} / 3$. The mean occupation numbers of particles, $\eta_{i}$, which are the packing fractions in zero dimension, are obtained from

$$
\eta_{i}=z_{i} \frac{\partial}{\partial z_{i}} \ln \Xi
$$

More explicitly,

$$
\begin{aligned}
& \eta_{\mathrm{c}}=\frac{z_{\mathrm{c}}}{z_{\mathrm{c}}+\exp \left(z_{\mathrm{p}}\right)}, \\
& \eta_{\mathrm{p}}=\frac{z_{\mathrm{p}} \exp \left(z_{\mathrm{p}}\right)}{z_{\mathrm{c}}+\exp \left(z_{\mathrm{p}}\right)},
\end{aligned}
$$

which can be inverted to obtain the fugacities

$$
\begin{aligned}
z_{\mathrm{c}} & =\frac{\eta_{\mathrm{c}}}{1-\eta_{\mathrm{c}}} \exp \left(\frac{\eta_{\mathrm{p}}}{1-\eta_{\mathrm{c}}}\right), \\
z_{\mathrm{p}} & =\frac{\eta_{\mathrm{p}}}{1-\eta_{\mathrm{c}}} .
\end{aligned}
$$

The dimensionless excess chemical potentials, $\tilde{\mu}_{i} \equiv \ln \left(z_{i}\right)-\ln \left(\eta_{i}\right)$, given by

$$
\begin{aligned}
& \tilde{\mu}_{\mathrm{c}}\left(\eta_{\mathrm{c}}, \eta_{\mathrm{p}}\right)=-\ln \left(1-\eta_{\mathrm{c}}\right)+\frac{\eta_{\mathrm{p}}}{1-\eta_{\mathrm{c}}}, \\
& \tilde{\mu}_{\mathrm{p}}\left(\eta_{\mathrm{c}}\right)=-\ln \left(1-\eta_{\mathrm{c}}\right),
\end{aligned}
$$

can then be used to integrate along a suitably chosen path in the $\left(\eta_{\mathrm{c}}, \eta_{\mathrm{p}}\right)$ plane in order to obtain the excess free energy

$$
\beta F_{0 \mathrm{~d}}\left(\eta_{\mathrm{c}}, \eta_{\mathrm{p}}\right)=\int_{0}^{\eta_{\mathrm{c}}} \mathrm{d} \eta_{c}^{\prime} \tilde{\mu}_{\mathrm{c}}\left(\eta_{c}^{\prime}, 0\right)+\int_{0}^{\eta_{\mathrm{p}}} \mathrm{d} \eta \tilde{\mu}_{\mathrm{p}}\left(\eta_{\mathrm{c}}\right)
$$

(Alternatively, one could use $\beta F_{0 \mathrm{~d}}=-\ln \Xi+\tilde{\mu}_{\mathrm{c}} \partial \ln \Xi / \partial \tilde{\mu}_{\mathrm{c}}+\tilde{\mu}_{\mathrm{p}} \partial \ln \Xi / \partial \tilde{\mu}_{\mathrm{p}}$.) The result is

$$
\beta F_{0 \mathrm{~d}}\left(\eta_{\mathrm{c}}, \eta_{\mathrm{p}}\right)=\left(1-\eta_{\mathrm{c}}-\eta_{\mathrm{p}}\right) \ln \left(1-\eta_{\mathrm{c}}\right)+\eta_{\mathrm{c}}
$$

This zero-dimensional free energy exhibits a number of physically realistic properties, that are generic to the model itself, independent of the precise situation under consideration, and which remain valid for other reduced dimensionalities and confining geometries:

(i) The colloid packing fraction is restricted to $\eta_{\mathrm{c}}<1$. In three dimensions, $\eta_{\mathrm{c}} \rightarrow 1$ corresponds to space-filling spheres. Although it is impossible to pack HS more densely than the close-packing fcc value of 0.7405 , the limit $\eta_{\mathrm{c}} \rightarrow 1$ does arise in many liquid state approximations such as scaled-particle and Percus-Yevick (PY) theories which commonly produce expressions containing factors of $1 /\left(1-\eta_{\mathrm{c}}\right)$.

(ii) Polymer packing fractions can be arbitrarily large, as no upper bound exists for $\eta_{\mathrm{p}}$; the ideality of the polymers means that there are no packing constraints for this species.

(iii) The free energy depends linearly on $\eta_{\mathrm{p}}$. As we shall show in section 2.6, this is not necessarily the case in three-dimensional bulk where terms of $\mathrm{O}\left(\eta_{\mathrm{p}}^{4}\right)$ and higher, but not $\mathrm{O}\left(\eta_{\mathrm{p}}^{2}\right)$ or $\mathrm{O}\left(\eta_{\mathrm{p}}^{3}\right)$, can arise. However, this simple linear dependence is a feature of the well known (approximate) free-volume theory $[4,5]$. 
Additionally, it is interesting to compare the present result for the AO model with the result for HS mixtures. For a binary HS mixture of species 1 and 2, one obtains $\beta F_{0 \mathrm{~d}}^{\mathrm{bhs}}=\left(1-\eta_{1}-\eta_{2}\right) \ln \left(1-\eta_{1}-\eta_{2}\right)+\eta_{1}+\eta_{2}$, where $\eta_{1}, \eta_{2}$ are the packing fractions of species 1 and 2 . Guided by (iii), we observe that linearization in $\eta_{2}$ yields $F_{0 \mathrm{~d}}$ of equation (14) upon identifying $\eta_{1}=\eta_{\mathrm{c}}$ and $\eta_{2}=\eta_{\mathrm{p}}$. This linearization property will be exploited further in section 2.6 in an alternative derivation of the DFT.

These two exact limiting cases provide the key building blocks for constructing the freeenergy functional.

\subsection{Density functional for the AO model}

Following previous work on HS mixtures [18-20], we express the excess Helmholtz free energy in terms of a functional of colloid and polymer density fields as a spatial integral over a free-energy density $\Phi$ that is a function of the weighted densities:

$$
\beta F_{\mathrm{exc}}\left[\rho_{\mathrm{c}}(\boldsymbol{r}), \rho_{\mathrm{p}}\left(\boldsymbol{r}^{\prime}\right)\right]=\int \mathrm{d}^{3} x \Phi\left(\left\{n_{v}^{\mathrm{c}}(\boldsymbol{x})\right\},\left\{n_{\gamma}^{\mathrm{p}}(\boldsymbol{x})\right\}\right),
$$

where the weighted densities for each species $i=\mathrm{c}, \mathrm{p}$ are obtained by convolutions with the actual density profiles

$$
n_{\nu}^{i}(\boldsymbol{x})=\int \mathrm{d}^{3} r \rho_{i}(\boldsymbol{r}) w_{\nu}^{i}(\boldsymbol{x}-\boldsymbol{r})
$$

In previous work on HS, $\Phi$ was taken to be a function of species-independent weighted densities [18]. Here we must generalize and allow $\Phi$ to depend on species-dependent weighted densities. This is necessary in order to capture the intrinsically different nature of colloids and polymers. The weight functions $w_{v}^{i}$ are independent of the density profiles and are given by

$$
\begin{aligned}
& w_{3}^{i}(\boldsymbol{r})=\Theta\left(R_{i}-r\right), \\
& w_{2}^{i}(\boldsymbol{r})=\delta\left(R_{i}-r\right), \\
& w_{1}^{i}(\boldsymbol{r})=\delta\left(R_{i}-r\right) /(4 \pi r), \\
& w_{0}^{i}(\boldsymbol{r})=\delta\left(R_{i}-r\right) /\left(4 \pi r^{2}\right), \\
& \boldsymbol{w}_{\mathrm{v} 2}^{i}(\boldsymbol{r})=\delta\left(R_{i}-r\right) \boldsymbol{r} / r, \\
& \boldsymbol{w}_{\mathrm{v} 1}^{i}(\boldsymbol{r})=\delta\left(R_{i}-r\right) \boldsymbol{r} /\left(4 \pi r^{2}\right), \\
& \hat{\boldsymbol{w}}_{\mathrm{m} 2}^{i}(\boldsymbol{r})=\delta\left(R_{i}-r\right)\left(\boldsymbol{r} \boldsymbol{r} / r^{2}-\hat{\mathbf{1}} / 3\right),
\end{aligned}
$$

where $r=|r|, \Theta(r)$ is the step function, $\delta(r)$ is the Dirac distribution and $\hat{\mathbf{1}}$ is the identity matrix. The weight functions are quantities with dimension length ${ }^{3-v}$. They differ in their tensorial rank: $w_{0}^{i}, w_{1}^{i}, w_{2}^{i}, w_{3}^{i}$ are scalars; $\boldsymbol{w}_{\mathrm{v} 1}^{i}, \boldsymbol{w}_{\mathrm{v} 2}^{i}$ are vectors; $\hat{\boldsymbol{w}}_{\mathrm{m} 2}^{i}$ is a (traceless) secondrank tensor. Equations (17)-(22) are the weights given in [18], whereas equation (23) is equivalent to the tensor formulation in [22]. The Fourier transforms of the weight functions are given in appendix A.

The free-energy density is composed of three parts

$$
\Phi=\Phi_{1}+\Phi_{2}+\Phi_{3},
$$

which are defined as

$$
\begin{aligned}
& \Phi_{1}=\sum_{i=\mathrm{c}, \mathrm{p}} n_{0}^{i} \varphi_{i}\left(n_{3}^{\mathrm{c}}, n_{3}^{\mathrm{p}}\right), \\
& \Phi_{2}=\sum_{i, j=\mathrm{c}, \mathrm{p}}\left(n_{1}^{i} n_{2}^{j}-\boldsymbol{n}_{\mathrm{v} 1}^{i} \cdot \boldsymbol{n}_{\mathrm{v} 2}^{j}\right) \varphi_{i j}\left(n_{3}^{\mathrm{c}}, n_{3}^{\mathrm{p}}\right),
\end{aligned}
$$


$\Phi_{3}=\frac{1}{8 \pi} \sum_{i, j, k=\mathrm{c}, \mathrm{p}}\left(\frac{1}{3} n_{2}^{i} n_{2}^{j} n_{2}^{k}-n_{2}^{i} \boldsymbol{n}_{\mathrm{v} 2}^{j} \cdot \boldsymbol{n}_{\mathrm{v} 2}^{k}+\frac{3}{2}\left[\boldsymbol{n}_{\mathrm{v} 2}^{i} \hat{\mathbf{n}}_{\mathrm{m} 2}^{j} \boldsymbol{n}_{\mathrm{v} 2}^{k}-\operatorname{tr}\left(\hat{\mathbf{n}}_{\mathrm{m} 2}^{i} \hat{\mathbf{n}}_{\mathrm{m} 2}^{j} \hat{\mathbf{n}}_{\mathrm{m} 2}^{k}\right)\right]\right) \varphi_{i j k}\left(n_{3}^{\mathrm{c}}, n_{3}^{\mathrm{p}}\right)$,

where tr denotes the trace, and derivatives of the zero-dimensional excess free energy (given by equation (14)) are

$$
\varphi_{i \ldots k}\left(\eta_{\mathrm{c}}, \eta_{\mathrm{p}}\right) \equiv \frac{\partial^{m}}{\partial \eta_{i} \ldots \partial \eta_{k}} \beta F_{0 \mathrm{~d}}\left(\eta_{\mathrm{c}}, \eta_{\mathrm{p}}\right)
$$

In the absence of polymer, $\Phi_{1}$ and $\Phi_{2}$ are equivalent to the free-energy densities for HS introduced in [18], and $\Phi_{3}$ is equivalent to the tensor treatment for pure HS in [22]. Equations (25)-(27) are direct generalizations of these earlier treatments to include summation over species. All the derivatives $\varphi_{i \ldots j}$ that carry more than one polymer index vanish due to the functional form of $F_{0 \mathrm{~d}}$, and we obtain

$$
\begin{aligned}
\Phi_{1} & =n_{0}^{\mathrm{c}}\left[-\ln \left(1-n_{3}^{\mathrm{c}}\right)+\frac{n_{3}^{\mathrm{p}}}{1-n_{3}^{\mathrm{c}}}\right]-n_{0}^{\mathrm{p}} \ln \left(1-n_{3}^{\mathrm{c}}\right), \\
\Phi_{2} & \left.=\left(n_{1}^{\mathrm{c}} n_{2}^{\mathrm{c}}-\boldsymbol{n}_{\mathrm{v} 1}^{\mathrm{c}} \cdot \boldsymbol{n}_{\mathrm{v} 2}^{\mathrm{c}}\right)\left[\frac{1}{1-n_{3}^{\mathrm{c}}}+\frac{n_{3}^{\mathrm{p}}}{\left(1-n_{3}^{\mathrm{c}}\right)^{2}}\right]+\frac{n_{1}^{\mathrm{p}} n_{2}^{\mathrm{c}}-\boldsymbol{n}_{\mathrm{v} 1}^{\mathrm{p}} \cdot \boldsymbol{n}_{\mathrm{v} 2}^{\mathrm{c}}+n_{1}^{\mathrm{c}} n_{2}^{\mathrm{p}}-\boldsymbol{n}_{\mathrm{v} 1}^{\mathrm{c}} \cdot \boldsymbol{n}_{\mathrm{v} 2}^{\mathrm{p}}}{1-n_{3}^{\mathrm{c}}},(30) \hat{\ln }_{\mathrm{m} 2}^{\mathrm{c}}\right) \\
\Phi_{3} & \left.=\frac{\frac{1}{3}\left(n_{2}^{\mathrm{c}}\right)^{3}-n_{2}^{\mathrm{c}}\left(\boldsymbol{n}_{\mathrm{v} 2}^{\mathrm{c}}\right)^{2}+\frac{3}{2}\left(\boldsymbol{n}_{\mathrm{v} 2}^{\mathrm{c}} \hat{\mathbf{n}}_{\mathrm{m} 2}^{\mathrm{c}} \boldsymbol{n}_{\mathrm{v} 2}^{\mathrm{c}}-3 \operatorname{det}\right.}{8 \pi}+\frac{2 n_{3}^{\mathrm{p}}}{\left(1-n_{3}^{\mathrm{c}}\right)^{2}}\right] \\
+ & \frac{\left(n_{2}^{\mathrm{c}}\right)^{2} n_{2}^{\mathrm{p}}-n_{2}^{\mathrm{p}}\left(\boldsymbol{n}_{\mathrm{v} 2}^{\mathrm{c}}\right)^{2}-2 n_{2}^{\mathrm{c}} \boldsymbol{n}_{\mathrm{v} 2}^{\mathrm{c}} \cdot \boldsymbol{n}_{\mathrm{v} 2}^{\mathrm{p}}+\frac{3}{2}\left\{2 \boldsymbol{n}_{\mathrm{v} 2}^{\mathrm{p}} \hat{\mathbf{n}}_{\mathrm{m} 2}^{\mathrm{c}} \boldsymbol{n}_{\mathrm{v} 2}^{\mathrm{c}}+\boldsymbol{n}_{\mathrm{v} 2}^{\mathrm{c}} \hat{\mathbf{n}}_{\mathrm{m} 2}^{\mathrm{p}} \boldsymbol{n}_{\mathrm{v} 2}^{\mathrm{c}}-3 \operatorname{tr}\left[\left(\hat{\mathbf{n}}_{\mathrm{m} 2}^{\mathrm{c}}\right)^{2} \hat{\mathbf{n}}_{\mathrm{m} 2}^{\mathrm{p}}\right]\right\}}{8 \pi\left(1-n_{3}^{\mathrm{c}}\right)^{2}} .
\end{aligned}
$$

For brevity we have omitted the $r$ dependence, e.g. $n_{3}^{\mathrm{c}}=n_{3}^{\mathrm{c}}(\boldsymbol{r})$. The DFT for the AO model is now fully specified. In the next subsection we examine several limiting cases in order to ascertain better the nature of the approximation we have introduced.

\subsection{Examining limiting cases of the functional}

2.5.1. Low density expansion. For low densities, $\rho_{i} \rightarrow 0$, the weighted densities also become small, $n_{v}^{i} \rightarrow 0$, since the latter are given by a convolution of the density with a density-independent weight function $w_{v}^{i}$, equation (16). In order to obtain the behaviour of the DF, we Taylor expand the free-energy density $\Phi$ in powers of weighted densities. The straightforward calculation yields to lowest order

$\Phi=n_{0}^{\mathrm{c}} n_{3}^{\mathrm{c}}+n_{1}^{\mathrm{c}} n_{2}^{\mathrm{c}}-\boldsymbol{n}_{\mathrm{v} 1}^{\mathrm{c}} \cdot \boldsymbol{n}_{\mathrm{v} 2}^{\mathrm{c}}+n_{0}^{\mathrm{c}} n_{3}^{\mathrm{p}}+n_{1}^{\mathrm{c}} n_{2}^{\mathrm{p}}-\boldsymbol{n}_{\mathrm{v} 1}^{\mathrm{c}} \cdot \boldsymbol{n}_{\mathrm{v} 2}^{\mathrm{p}}+n_{0}^{\mathrm{p}} n_{3}^{\mathrm{c}}+n_{1}^{\mathrm{p}} n_{2}^{\mathrm{c}}-\boldsymbol{n}_{\mathrm{v} 1}^{\mathrm{p}} \cdot \boldsymbol{n}_{\mathrm{v} 2}^{\mathrm{c}}$.

Each of the terms in the sum is a bilinear combination of weighted densities, $n_{\nu}^{i} n_{\lambda}^{j}$. We rewrite this formally as

$$
\Phi \equiv \sum_{i j=\mathrm{cp}} \sum_{\nu \lambda} C_{\nu \lambda}^{i j} n_{\nu}^{i} n_{\lambda}^{j}
$$

where the coefficients take on values $C_{\nu \lambda}^{i j}=-1,0,1$. (Note that the coefficients $C_{v \lambda}^{\mathrm{pp}}$ of terms $n_{\nu}^{\mathrm{p}} n_{\lambda}^{\mathrm{p}}$, with $\mathrm{p}$ representing species 2 , which are non-zero in the corresponding expansion for the binary HS functional vanish in the AO model.) Next we rearrange the order of integrations in the density functional

$\beta F_{\text {exc }}=\int \mathrm{d}^{3} x \sum_{i j \nu \lambda} C_{\nu \lambda}^{i j} \int \mathrm{d}^{3} r \rho_{i}(\boldsymbol{r}) w_{\nu}^{i}(\boldsymbol{r}-\boldsymbol{x}) \int \mathrm{d}^{3} r^{\prime} \rho_{j}\left(\boldsymbol{r}^{\prime}\right) w_{\lambda}^{j}\left(\boldsymbol{r}^{\prime}-\boldsymbol{x}\right)$ 


$$
\begin{aligned}
& =\sum_{i j} \int \mathrm{d}^{3} r \rho_{i}(\boldsymbol{r}) \int \mathrm{d}^{3} r^{\prime} \rho_{j}\left(\boldsymbol{r}^{\prime}\right) \sum_{\nu \lambda} \int \mathrm{d}^{3} x C_{\nu \lambda}^{i j} w_{\nu}^{i}(\boldsymbol{r}-\boldsymbol{x}) w_{\lambda}^{j}\left(\boldsymbol{r}^{\prime}-\boldsymbol{x}\right) \\
& =\sum_{i j} \int \mathrm{d}^{3} r \rho_{i}(\boldsymbol{r}) \int \mathrm{d}^{3} r^{\prime} \rho_{j}\left(\boldsymbol{r}^{\prime}\right) f_{i j}\left(\left|\boldsymbol{r}-\boldsymbol{r}^{\prime}\right|\right) / 2,
\end{aligned}
$$

where in the last step we exploited the property of the weight functions to obtain the HS Mayer bond. This is the same argument as in Rosenfeld's HS mixture case [18]; the vanishing of the polymer-polymer Mayer bond is due solely to the functional form of $\Phi$, which does not contain terms quadratic in polymer weighted densities. Thus the present functional does reduce to the correct low density limit when all densities $\rho_{i} \rightarrow 0$.

2.5.2. Zero-dimensional limit. For density distributions $\rho_{i}=\eta_{i} \delta(\boldsymbol{r})$ each weighted density becomes proportional to its weight function

$$
n_{\nu}^{i}(\boldsymbol{x})=\int \mathrm{d}^{3} r \eta_{i} \delta(\boldsymbol{r}) w_{\nu}^{i}(\boldsymbol{r}-\boldsymbol{x})=\eta_{i} w_{\nu}^{i}(\boldsymbol{x}) .
$$

We show that the contribution from $\Phi_{1}$ yields the exact result. Consider

$$
\begin{aligned}
\beta F_{\mathrm{exc}} & =\int \mathrm{d}^{3} r \Phi_{1} \\
& =\int_{0}^{\infty} \mathrm{d} r 4 \pi r^{2} \sum_{i} \eta_{i} w_{0}^{i}(r) \varphi_{i}\left(\eta_{\mathrm{c}} w_{3}^{\mathrm{c}}(r), \eta_{\mathrm{p}} w_{3}^{\mathrm{p}}(r)\right) \\
& =\int_{0}^{\infty} \mathrm{d} r \sum_{i} \eta_{i} \delta\left(R_{i}-r\right) \varphi_{i}\left(\eta_{\mathrm{c}} \Theta\left(R_{\mathrm{c}}-r\right), \eta_{\mathrm{p}} \Theta\left(R_{\mathrm{p}}-r\right)\right) \\
& =\int_{0}^{\infty} \mathrm{d} r \sum_{i} \eta_{i} \frac{-\mathrm{d} \Theta\left(R_{i}-r\right)}{\mathrm{d} r} \varphi_{i}\left(\eta_{\mathrm{c}} \Theta\left(R_{\mathrm{c}}-r\right), \eta_{\mathrm{p}} \Theta\left(R_{\mathrm{p}}-r\right)\right) \\
& =\int_{0}^{1} \mathrm{~d} t\left[\eta_{\mathrm{p}} \varphi_{\mathrm{p}}\left(\eta_{\mathrm{c}}, \eta_{\mathrm{p}} t\right)+\eta_{\mathrm{c}} \varphi_{\mathrm{c}}\left(\eta_{\mathrm{c}} t, 0\right)\right], \\
& =\beta F_{0 \mathrm{~d}}\left(\eta_{\mathrm{c}}, \eta_{\mathrm{p}}\right)
\end{aligned}
$$

where we have assumed, without loss of generality, in equation (42) that $R_{\mathrm{c}}>R_{\mathrm{p}}$ and have used the definition of $\varphi_{i}$, equation (28), in the last step.

The contributions from $\Phi_{2}$ and $\Phi_{3}$ both vanish. This follows from symmetry considerations. From equation (37), and the fact that $\boldsymbol{w}_{\mathrm{v} v}^{i}=w_{\nu}^{i} \boldsymbol{r} / r$ for $v=1,2$, it follows that $n_{1}^{i} n_{2}^{j}=\boldsymbol{n}_{\mathrm{v} 1}^{i} \cdot \boldsymbol{n}_{\mathrm{v} 2}^{j}$. Hence, from equation (30), $\Phi_{2}=0$. A similar argument holds for $\Phi_{3}$.

2.5.3. One-dimensional limit. Following [21] we can obtain an effective one-dimensional functional by imposing on the general three-dimensional functional density distributions $\rho_{i}(\boldsymbol{r})=\rho_{i}(x) \delta(y) \delta(z)$. We omit the tedious details and show the final result:

$$
\beta F_{\mathrm{exc}}\left[\rho_{\mathrm{c}}(x), \rho_{\mathrm{p}}\left(x^{\prime}\right)\right]=\int \mathrm{d} x \Phi\left(\left\{n_{v}^{\mathrm{c}}(x)\right\},\left\{n_{\gamma}^{\mathrm{p}}(x)\right\}\right),
$$

where the weighted densities are obtained by one-dimensional convolutions

$$
n_{v}^{i}(x)=\int \mathrm{d} x^{\prime} \rho_{i}\left(x^{\prime}\right) w_{v}^{i}\left(x-x^{\prime}\right),
$$

of the two one-dimensional weight functions

$$
\begin{aligned}
& w_{d}^{i}(x)=\theta\left(R_{i}-|x|\right), \\
& w_{0}^{i}(x)=\left[\delta\left(R_{i}+x\right)+\delta\left(R_{i}-x\right)\right] / 2 .
\end{aligned}
$$


Note that $w_{d}^{i}$ originates from the three-dimensional weight $w_{3}^{i}$, and describes the onedimensional packing fraction. The excess free-energy density obtained from $\Phi_{1}+\Phi_{2}$, i.e. equations (29), (30), is

$$
\begin{aligned}
\Phi & =\sum_{i=\mathrm{c}, \mathrm{p}} n_{0}^{i} \varphi_{i}\left(n_{d}^{\mathrm{c}}, n_{d}^{\mathrm{p}}\right) \\
& =-\left(n_{0}^{\mathrm{c}}+n_{0}^{\mathrm{p}}\right) \ln \left(1-n_{d}^{\mathrm{c}}\right)+\frac{n_{0}^{\mathrm{c}} n_{d}^{\mathrm{p}}}{1-n_{d}^{\mathrm{c}}} .
\end{aligned}
$$

Note that the remaining term $\Phi_{3}$, equation (31), gives an additional contribution if $q \neq 1$. Following the discussion of two-dimensional crossover in HS mixtures [35] we expect this contribution to be negligible. We note that the bulk equation of state derived from $\Phi$ in equation (49) is the same as that of free-volume theory in one dimension. As the latter is known to yield a very good approximation to the exact solution of the one-dimensional AO model [36], provided the reservoir packing fraction $\eta_{\mathrm{p}, \mathrm{r}}$ is smaller than about 1.5 , it is likely that the one-dimensional functional will also be accurate in this regime.

To summarize, in this subsection we have shown that our DF for the AO model does yield the correct low density and zero-dimensional limits and that for uniform one-dimensional density distributions the theory is equivalent to free-volume theory. For completeness, a twodimensional functional is presented in appendix B.

\subsection{Alternative derivation of the DFT}

We show here that the present functional (section 2.4) can be derived in an alternative fashion starting from the binary HS functional of Rosenfeld [18]. The only distinction between the $\mathrm{AO}$ model and the binary HS model is that the interaction $V_{22}$ between particles of species 2 is zero. Thus all the necessary geometrical information about sphere packing is already included and it is possible, at least in principle, to extract a functional for the AO model. More precisely, if we had the exact functional for the binary HS model then a density expansion would contain all the diagrams required for the AO model plus an additional class of diagrams containing Mayer bonds between polymers. An operation which removes the unwanted class diagrams from the binary HS functional would yield the exact functional for the AO model. In order to extract the information we require from the binary HS functional, we turn to the exact low density diagrammatic expansion of the bulk pair direct correlation function. For the AO model this is given by

$$
\begin{aligned}
& c_{\mathrm{cc}}^{(2)}(r)=o_{\mathrm{c}} \mathrm{O}_{\mathrm{c}}+\rho_{c} \\
& c_{\mathrm{cp}}^{(2)}(r)=0_{\overline{\mathrm{c}}} \mathrm{O}_{\mathrm{p}}+\rho_{c} \quad \rho_{\mathrm{c}} \quad \overbrace{\mathrm{c}}^{\mathrm{c}}+\mathcal{O}\left(\rho^{2}\right) \\
& c_{\mathrm{pp}}^{(2)}(r)=0+\mathcal{O}\left(\rho^{2}\right),
\end{aligned}
$$

where we have given the expansion up to second order (third virial level). As usual each bond between circles represents a Mayer function and a shaded circle an integration over one coordinate. All diagrams involving a polymer-polymer Mayer bond are zero. To third virial level, $c_{\mathrm{pp}}^{(2)}=0$, as all three contributing diagrams contain a polymer-polymer Mayer bond. Higher order terms in the expansion of $c_{\mathrm{pp}}^{(2)}$ are non-zero as there exist diagrams which contribute and yet do not contain polymer-polymer Mayer bonds. For example, at the fourth virial level the diagram

$$
\overbrace{p}^{c}
$$


appears and gives a contribution of order $\rho_{p}^{2}$ to $c_{\mathrm{pp}}^{(2)}(r)$. It should be noted that the PercusYevick theory for the binary AO model also gives $c_{\mathrm{pp}}^{(2)}=0$. A theory capable of going beyond the approximation $c_{\mathrm{pp}}^{(2)}=0$ would have to be accurate to at least the fourth virial level. As even sophisticated integral equation theories are usually exact to only third virial level this is clearly a difficult task. Equations (50)-(52) give no information about how to extract the colloidpolymer functional from the binary HS functional as information about the polymer-polymer interaction does not enter until higher terms in the expansion. However, the facts that

(i) $c_{\mathrm{cp}}^{(2)}$ is independent of the polymer density field and

(ii) $c_{\mathrm{pp}}^{(2)}=0$ suggest that the simplest way to obtain a functional for the AO model is to linearize the binary HS functional with respect to $\rho_{p}(\boldsymbol{r})$.

This ensures that the bulk pair correlations are correct to the third virial level, i.e. $c_{\mathrm{pp}}^{(2)}=$ $-\delta^{2} \beta F_{\text {exc }} / \delta \rho_{\mathrm{p}}(\boldsymbol{r}) \delta \rho_{\mathrm{p}}\left(\boldsymbol{r}^{\prime}\right)=0$ and $c_{\mathrm{cp}}^{(2)}=-\delta^{2} \beta F_{\mathrm{exc}} / \delta \rho_{\mathrm{c}}(\boldsymbol{r}) \delta \rho_{\mathrm{p}}\left(\boldsymbol{r}^{\prime}\right)$ is independent of the polymer density.

The exact AO free-energy functional contains terms linear in $\rho_{p}(\boldsymbol{r})$ but not terms in $\rho_{p}^{2}$ or $\rho_{p}^{3}$, as these would lead to inconsistency with the low order diagrams. However, when expanding the binary HS functional in $\rho_{p}(\boldsymbol{r})$ we could have chosen to omit the $\rho_{p}^{2}$ and $\rho_{p}^{3}$ terms, but retain all higher orders; the low order diagrams exercise no constraint over these higher order contributions. In linearizing the functional we have chosen to set all higher order terms to zero. The justification for this can be found by looking at the exact diagrammatic expansion for a binary mixture to fourth virial level:

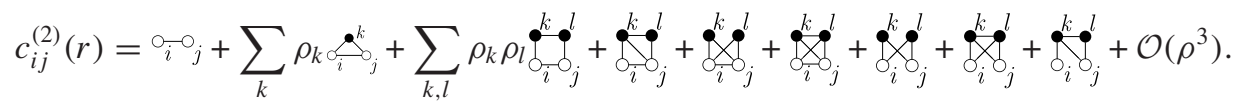

The Rosenfeld binary HS functional generates bulk $c_{i j}^{(2)}$ identical to those from Percus-Yevick theory. The latter is not exact to fourth virial level as it contains only a subset of the required diagrams:

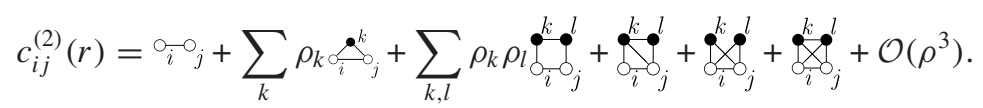

All diagrams in PY theory, equation (55), have a Mayer bond connecting $i j$. We henceforth refer to diagrams of this category as A class and diagrams with no $i j$ Mayer bond as B class and focus our attention on $c_{\mathrm{pp}}^{(2)}$. Retaining quartic and higher order terms when expanding the Rosenfeld HS functional, only HS A-class diagrams would be included. For the AO model all A-class diagrams are identically zero in the expansion of $c_{\mathrm{pp}}^{(2)}$ and only B-class diagrams contribute. Inclusion of quartic or higher order terms in the $\rho_{p}$ expansion of the functional would incorporate only unphysical diagrams. The A-class diagrams required for the AO model are not contained within the original binary HS functional. We conclude that by linearizing the original Rosenfeld HS functional in the polymer density we should obtain a functional which describes the AO model. Indeed when such a linearization is performed we recover equations (24)-(31) for the free-energy density but with the tensor weighted densities $\hat{\mathbf{n}}_{\mathrm{m} 2}^{i}=0$, since the original Rosenfeld functional does not have tensor weights.

\subsection{Effective one-component functional}

When investigating the statistical mechanics of soft matter systems it is frequently advantageous to integrate out some degrees of freedom in order to obtain effective interactions between the particles of the remaining species. This has proved particularly fruitful for the AO 
model of colloid-polymer mixtures where the polymer degrees of freedom could be explicitly integrated out for certain size ratios $[5,8,9,13]$. However, it is not obvious that an analogous procedure can generally be performed within the context of DFT. In order to minimize a functional $\Omega$ of two independent density fields it is necessary to solve simultaneously the coupled equations

$$
\frac{\delta \Omega\left[\rho_{\mathrm{c}}, \rho_{\mathrm{p}}\right]}{\delta \rho_{\mathrm{c}}(\boldsymbol{r})}=0, \quad \frac{\delta \Omega\left[\rho_{\mathrm{c}}, \rho_{\mathrm{p}}\right]}{\delta \rho_{\mathrm{p}}(\boldsymbol{r})}=0 .
$$

The corresponding Euler-Lagrange equations are given by

$$
\begin{aligned}
& \rho_{\mathrm{c}}(\boldsymbol{r})=z_{\mathrm{c}} \exp \left(c_{\mathrm{c}}^{(1)}\left(\boldsymbol{r} ;\left[\rho_{\mathrm{c}}, \rho_{\mathrm{p}}\right]\right)-\beta V_{\mathrm{c}}^{\mathrm{ext}}(\boldsymbol{r})\right) \\
& \rho_{\mathrm{p}}(\boldsymbol{r})=z_{\mathrm{p}} \exp \left(c_{\mathrm{p}}^{(1)}\left(\boldsymbol{r} ;\left[\rho_{\mathrm{c}}, \rho_{\mathrm{p}}\right]\right)-\beta V_{\mathrm{p}}^{\operatorname{ext}}(\boldsymbol{r})\right),
\end{aligned}
$$

where $z_{c}$ and $z_{p}$ are the fugacities and the functional dependence of the one-body direct correlation function $c_{i}^{(1)}$ is made explicit. While species have been labelled c, p, equations (57) and (58) apply to an arbitrary binary mixture. The Euler-Lagrange equations are usually coupled in a complicated way and must be solved using numerical methods.

Within the context of DFT the term 'integrating out' can be somewhat misleading because at no stage in the calculation have integrals been performed over the polymer degrees of freedom. The usual procedure of integrating out is performed directly on the partition function and the effective potentials which result are then input to a one-component theory or simulation to calculate the density distribution of the species of (main) interest $[5,8]$. While an effective potential never appears explicitly within the DFT formulation, it is contained implicitly in the Euler-Lagrange equation of the species of interest. It is the variational minimization of $\Omega$ which takes the place of an explicit integrating out. The analogue of integrating out within DFT is expressing the free-energy functional as a functional of a reduced set of density fields

$$
F_{\mathrm{exc}}\left[\rho_{\mathrm{c}}(\boldsymbol{r})\right] \equiv F_{\mathrm{exc}}\left[\rho_{\mathrm{c}}(\boldsymbol{r}), \rho_{\mathrm{p}}\left[\rho_{\mathrm{c}}(\boldsymbol{r})\right]\right]
$$

These considerations can be made explicit for the AO functional. The one-body direct correlation function of species $i$ is given by

$$
c_{i}^{(1)}(\boldsymbol{r})=-\beta \frac{\delta F_{\mathrm{exc}}\left[\left\{\rho_{i}\right\}\right]}{\delta \rho_{i}(\boldsymbol{r})}=-\sum_{v} \frac{\partial \Phi}{\partial n_{v}^{i}} \otimes \omega_{v}^{i},
$$

where $\otimes$ denotes a convolution. $c_{i}^{(1)}(\boldsymbol{r})$ is independent of the polymer density as only colloid weighted densities appear on the RHS of equation (60). It follows that $c_{p}^{(1)}(\boldsymbol{r})$ is a functional of the colloid profile alone: $c_{p}^{(1)}(\boldsymbol{r}) \equiv c_{p}^{(1)}\left(\boldsymbol{r} ;\left[\rho_{c}\right]\right)$. Combining (60) with (58) thus provides an explicit expression for the polymer profile $\rho_{p}(\boldsymbol{r})$ as a functional of the colloid profile $\rho_{c}(\boldsymbol{r})$ [9]. If we constrain the colloid density field $\rho_{c}(\boldsymbol{r})$ to be fixed at some non-equilibrium value, then the polymer profile which minimizes the free energy subject to the constraint is automatically given. The AO functional, although a genuine two-component theory, can thus be regarded as an effective one-component free-energy functional of a single density field. For more general model fluids the Euler-Lagrange equations will not decouple and will not be as simple but the concept remains the same. Although the equations may be coupled in a complicated way, for given external potentials, $\rho_{1} \equiv \rho_{1}\left(\left[\rho_{2}\right] ; z_{1}\right)$ and $\rho_{2} \equiv \rho_{2}\left(\left[\rho_{1}\right] ; z_{2}\right)$ so one of the density profiles can, in principle, be eliminated to obtain the effective functional [37]. In order to obtain explicit forms for the effective potentials one must resort to alternative methods such as those described by Roth et al [38] for additive and non-additive [39] HS mixtures.

\section{Application to bulk fluid phases}

In this section we apply the theory developed in section 2 to the calculation of the thermodynamic and structural properties of the bulk AO mixture. 


\subsection{Thermodynamic functions}

Homogeneous fluid phases are characterized by spatially constant density fields: $\rho_{\mathrm{c}}(\boldsymbol{r}) \equiv \rho_{\mathrm{c}}$, and $\rho_{\mathrm{p}}(\boldsymbol{r}) \equiv \rho_{\mathrm{p}}$. In order to determine the free energy, and hence all thermodynamic quantities, we evaluate the density functional $F_{\mathrm{exc}}\left[\rho_{\mathrm{c}}=\right.$ const, $\rho_{\mathrm{p}}=$ const $]$. First we need to calculate the weighted densities, equation (16). The integration over the weight functions (17-23) can be carried out explicitly, and yields the so-called fundamental measures

$$
\xi_{v}^{i} \equiv \int \mathrm{d}^{3} x w_{v}^{i}(\boldsymbol{x})
$$

which describe the volume $\xi_{3}^{i}=4 \pi R_{i}^{3} / 3$, surface area $\xi_{2}^{i}=4 \pi R_{i}^{2}$, integral mean curvature $\xi_{1}^{i}=R_{i}$ and Euler characteristic $\xi_{0}^{i}=1$ of the spherical particles. The resulting weighted densities are

$$
\begin{aligned}
& n_{3}^{i}=\eta_{i}, \\
& n_{2}^{i}=3 \eta_{i} / R_{i}, \\
& n_{1}^{i}=3 \eta_{i} /\left(4 \pi R_{i}^{2}\right), \\
& n_{0}^{i}=3 \eta_{i} /\left(4 \pi R_{i}^{3}\right), \\
& \boldsymbol{n}_{\mathrm{v} 1}^{i}=\boldsymbol{n}_{\mathrm{v} 2}^{i}=\hat{\mathbf{n}}_{\mathrm{m} 2}^{i}=0,
\end{aligned}
$$

with, once again, $i=\mathrm{c}$, p. Inserting these expressions into equations (29)-(31), we obtain the excess free-energy density $\Phi$, equation (24), which is constant in space, so the integration in equation (15) is trivial. We find that the excess Helmholtz free-energy density is given by

$$
\beta F_{\text {exc }} / V=\beta f_{\mathrm{hs}}\left(\rho_{\mathrm{c}}\right)-\rho_{\mathrm{p}} \ln \alpha\left(\rho_{\mathrm{c}}\right),
$$

where $f_{\mathrm{hs}}\left(\rho_{\mathrm{c}}\right)$ is the excess free-energy density of pure HS in the scaled-particle (PY compressibility) approximation, given as

$$
\beta f_{\mathrm{hs}}\left(\rho_{\mathrm{c}}\right)=\frac{3 \eta_{\mathrm{c}}\left[3 \eta_{\mathrm{c}}\left(2-\eta_{\mathrm{c}}\right)-2\left(1-\eta_{\mathrm{c}}\right)^{2} \ln \left(1-\eta_{\mathrm{c}}\right)\right]}{8 \pi R_{\mathrm{c}}^{3}\left(1-\eta_{\mathrm{c}}\right)^{2}},
$$

and

$$
\alpha\left(\rho_{\mathrm{c}}\right)=\left(1-\eta_{\mathrm{c}}\right) \exp \left(-A \gamma-B \gamma^{2}-C \gamma^{3}\right),
$$

where $\gamma=\eta_{\mathrm{c}} /\left(1-\eta_{\mathrm{c}}\right), A=q^{3}+3 q^{2}+3 q, B=3 q^{3}+9 q^{2} / 2$ and $C=3 q^{3}$. This result can be shown to be identical to that of free-volume theory for the AO model [4], where the quantity $\alpha$ is interpreted as the ratio of the free volume accessible to a test polymer sphere and the system volume. In order to demonstrate the equivalence we perform a Legendre transform on the total (canonical) Helmholtz free energy $F\left(N_{\mathrm{c}}, N_{\mathrm{p}}, V\right)=F_{\text {exc }}\left(N_{\mathrm{c}}, N_{\mathrm{p}}, V\right)+$ $\beta^{-1} V \sum_{i=\mathrm{c}, \mathrm{p}} \rho_{i}\left[\ln \left(\Lambda_{i}^{3} \rho_{i}\right)-1\right]$ to obtain the semigrand potential

$$
\tilde{\Omega}\left(N_{\mathrm{c}}, z_{\mathrm{p}}, V\right)=F\left(N_{\mathrm{c}}, N_{\mathrm{p}}, V\right)-\mu_{\mathrm{p}} N_{\mathrm{p}} .
$$

The polymer chemical potential $\mu_{\mathrm{p}}=\left(\partial F / \partial N_{\mathrm{p}}\right)_{N_{\mathrm{c}}, V}=\beta^{-1} \ln \left(\Lambda_{\mathrm{p}}^{3} \rho_{\mathrm{p}} / \alpha\right)$ and equation (70) reduces to

$$
\frac{\beta \tilde{\Omega}\left(N_{\mathrm{c}}, z_{\mathrm{p}}, V\right)}{V}=\rho_{\mathrm{c}}\left[\ln \left(\Lambda_{\mathrm{c}}^{3} \rho_{\mathrm{c}}\right)-1\right]+\beta f_{\mathrm{hs}}\left(\rho_{\mathrm{c}}\right)-\rho_{\mathrm{p}}\left(\rho_{\mathrm{c}}, z_{\mathrm{p}}\right),
$$

where the polymer density in the system, $\rho_{\mathrm{p}}$, depends on the colloid density $\rho_{\mathrm{c}}$ and on the polymer fugacity $z_{\mathrm{p}}=\exp \left(\beta \mu_{\mathrm{p}}\right) / \Lambda_{\mathrm{p}}^{3}$. For ideal polymers $z_{\mathrm{p}}=\rho_{\mathrm{p}, \mathrm{r}}$, the density of polymer in the reservoir is in chemical equilibrium with the system. Equating the two expressions for $\mu_{\mathrm{p}}$ yields

$$
\rho_{\mathrm{p}}\left(\rho_{\mathrm{c}}, z_{\mathrm{p}}\right)=\alpha\left(\rho_{\mathrm{c}}\right) \rho_{\mathrm{p}, \mathrm{r}}=\alpha\left(\rho_{\mathrm{c}}\right) z_{\mathrm{p}},
$$


with $\alpha$ given by equation (69). Inserting equation (72) into (71) leads to the standard free-volume result for the semi-grand potential density [4]. Note that $-V^{-1} \partial \tilde{\Omega} / \partial \mu_{\mathrm{p}}=\rho_{\mathrm{p}}$, consistent with the thermodynamic definition of $\tilde{\Omega}$.

At first sight it seems surprising that our DFT approach is equivalent to free-volume theory. Recall that the latter treats the semi-grand potential as the sum of a HS (colloid) part plus a contribution from an ideal gas of polymers in the free volume left by the colloids. The connections between the two approaches become clearer when we recognize the following.

(i) The treatment of HSs is equivalent; $\alpha$ in free-volume theory is obtained from scaledparticle theory, while the DFT gives rise to the scaled particle equation of state for pure and binary HS mixtures.

(ii) Free-volume theory can be regarded as an expansion of $\tilde{\Omega}$ in the fugacity $z_{\mathrm{p}}$, about an HS reference system, that is truncated at the term linear in $z_{\mathrm{p}}[5,13]$. The linearity in $z_{\mathrm{p}}$, or equivalently in $\eta_{\mathrm{p}}$, is a key feature of our DFT which we imposed at the outset via the zero-dimensional route and in the alternative derivation of section 2.6. Note that once one has identified $\alpha$ as the ratio of polymer density in the system to that in the reservoir, see equation (72), its interpretation as the free-volume fraction for a single ideal polymer is immediate. That the DFT should yield the same formula for $\alpha$ as that of scaled-particle theory is then not so surprising. Nevertheless, it is pleasing that the two approaches, which appear to have rather distinct roots, do yield the same bulk free energy and hence the same (fluid) equation of state ${ }^{4}$. In particular they yield the same attractive contribution, $-\alpha\left(\rho_{\mathrm{c}}\right) z_{\mathrm{p}}$, to the grand potential density. It is this term which leads to the possibility of fluid-fluid demixing.

\subsection{Fluid-fluid demixing}

Phase behaviour in the AO model is a well studied problem [2-5]. It is striking that this simple model gives rise to stable, entropically driven fluid-fluid phase separation for sufficiently large size ratios $q$. Given that the free energy from our approach is identical to that from free-volume theory, it immediately follows that it gives rise to the same (fluid state) phase diagrams. We do not consider solid states in the present study. The phase separation into colloid-rich (polymerpoor) and colloid-poor (polymer-rich) fluid phases is analogous to liquid-gas separation with the polymer reservoir fraction $\eta_{\mathrm{p}, \mathrm{r}}$, or $z_{\mathrm{p}}$, playing the role of inverse temperature. Free-volume theory for the AO model predicts stable liquid-gas coexistence for $q>0.32$. For smaller values of $q$ this transition becomes metastable with respect to a broad, in $\eta_{\mathrm{c}}$, fluid-solid transition $[4,5]$. Here we focus on demixing for size ratios in the range $0.4 \leqslant q \leqslant 1$, where we expect stable liquid-gas coexistence.

In order to calculate the binodal we perform a common-tangent construction on the semigrand potential $\tilde{\Omega}$, equation (71), at fixed $z_{\mathrm{p}}$ (or $\eta_{\mathrm{p}, \mathrm{r}}$ ). This is equivalent to equating the total pressure and chemical potentials of each species in the two coexisting phases. It should be noted that the canonical free energy $F$ does not display any obvious double-minimum structure as a function of $\eta_{\mathrm{c}}, \eta_{\mathrm{p}}$. The spinodal is the locus of statepoints where the curvature of the semi-grand potential changes its sign at fixed $\eta_{\mathrm{p}, \mathrm{r}}$. In practice, a canonical calculation can be performed more easily. The boundary of stability is obtained by solving $\operatorname{det}\left[\partial^{2}(F / V) / \partial \rho_{i} \partial \rho_{j}\right]=0$, $i, j=\mathrm{c}, \mathrm{p}$. This can be done analytically, and we obtain the spinodal from the following equation:

${ }^{4}$ We note that in calculations, e.g. [5], based on free-volume theory the Carnahan-Starling approximation is often used for $f_{\mathrm{hs}}$ whereas in the DFT approach the PY compressibility approximation (68) must be employed. 

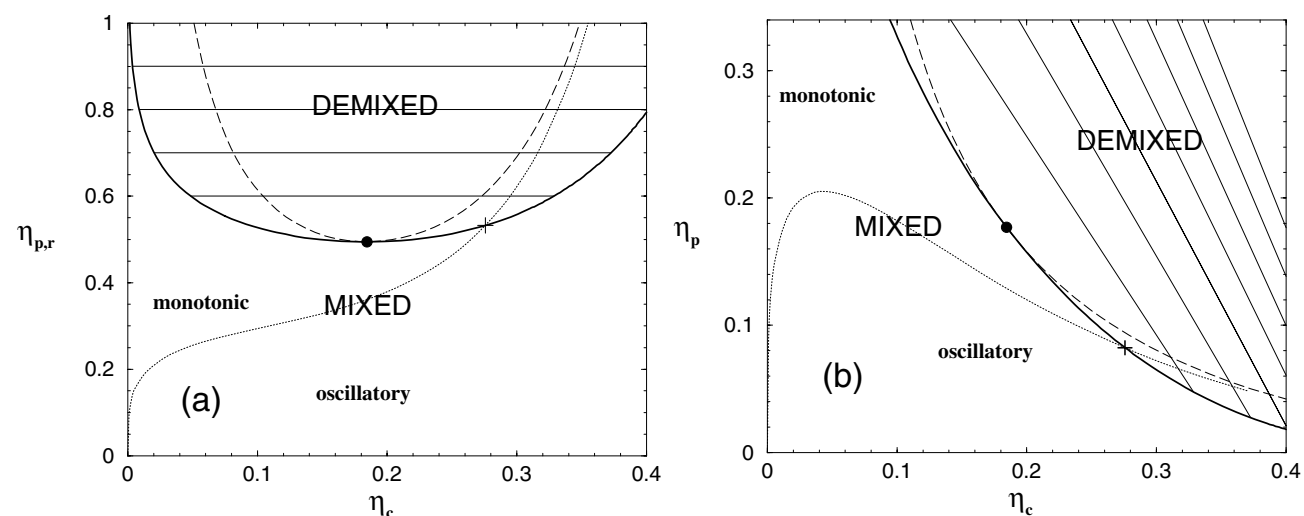

Figure 1. Demixing phase diagram of the AO model for size ratio $q=0.6$. The binodal (thick curve), spinodal (dashed curve), tie lines (thin curves), FW line (dotted curve), critical point (dot) and intersection of the binodal and the FW line (cross) are shown for (a) the reservoir representation using the colloid packing fraction $\eta_{\mathrm{c}}$ and polymer reservoir fraction $\eta_{\mathrm{p}, \mathrm{r}}$ as variables and (b) the system representation using $\eta_{\mathrm{c}}$ and the actual polymer fraction $\eta_{\mathrm{p}}$ as variables. The FW line denotes the line where the asymptotic decay of pair correlations crosses over from monotonic to oscillatory. Note that these phase diagrams display only the fluid portion of the phase diagram; a fluid-solid transition occurs for higher values of $\eta_{\mathrm{c}}$.

$$
\eta_{\mathrm{p}}=\frac{\theta_{1}^{4} \theta_{2} / \eta_{\mathrm{c}}}{12 \theta_{1}^{3}+15 q \theta_{1}^{2} \theta_{2}+6 q^{2} \theta_{1} \theta_{2}^{2}+q^{3} \theta_{2}^{3}},
$$

where $\theta_{1}=1-\eta_{\mathrm{c}}$, and $\theta_{2}=1+2 \eta_{\mathrm{c}}$. Results obtained in one representation are easily converted to the other using equation (72).

In figure 1 we display the demixing phase diagram for size ratio $q=0.6$. In the reservoir representation (figure 1(a)), we see that the form of the phase diagram does resemble the gas-liquid portion of the phase diagram of a simple substance provided the polymer reservoir density $\eta_{\mathrm{p}, \mathrm{r}}$ is replaced by inverse temperature. Upon increasing $\eta_{\mathrm{p}, \mathrm{r}}$ the system separates into phases with different compositions and the tie lines connecting coexisting phases are horizontal in this representation. The binodal and spinodal coincide at the (lower) critical point. Also shown in figure 1 is the FW line, which divides the phase diagram into regions where the asymptotic decay of pair correlations is either monotonic or oscillatory. We shall discuss this in detail in section 3.5 .

The actual polymer density in the system may be considerably different from that in the reservoir, because insertion of polymers into the system is hindered by the presence of the colloids, i.e. $\eta_{\mathrm{p}}<\eta_{\mathrm{p}, \mathrm{r}}$. The phase diagram in the system representation (figure 1(b)) shows the mixed one-phase region in the lower left part and the demixed two-phase region in the upper right part of the $\eta_{\mathrm{c}}, \eta_{\mathrm{p}}$ plane. The tie lines between coexisting phases now have a negative slope. Changing the size ratio $q$ has a pronounced effect on the location of the binodal. We display results for $q=0.4,0.6,0.8,1$ in figure 2 . As $q$ increases, the binodals move to higher $\eta_{\mathrm{p}, \mathrm{r}}$, and the critical point shifts to lower colloid fractions $\eta_{\mathrm{c}}$ (see figure 2(a)). The actual polymer density $\eta_{\mathrm{p}}$ at the critical point increases slowly with $q$ (figure 2(b)).

\subsection{Direct correlation functions}

We now shift our attention to the structural properties of the bulk AO mixture, focusing first on the pair direct correlation functions $c_{i j}^{(2)}(r)$. Recall that for binary HS mixtures the 

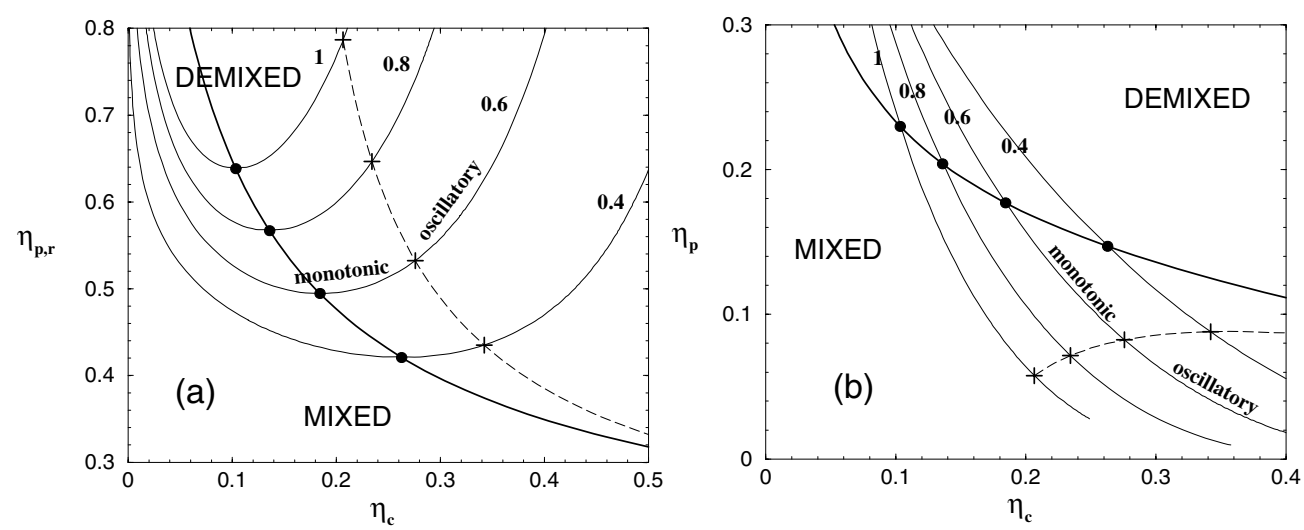

Figure 2. Phase diagram of the $\mathrm{AO}$ model for size ratios $q=0.4,0.6,0.8,1$. The binodals (thin curves), critical points (dots joined by a thick curve) and intersections of the FW line and the binodals (crosses joined by a dashed curve) are shown for (a) the reservoir representation with $\eta_{\mathrm{c}}$ and $\eta_{\mathrm{p}, \mathrm{r}}$ and (b) the system representation with $\eta_{\mathrm{c}}$ and $\eta_{\mathrm{p}}$.

$c_{i j}^{(2)}(r)$ obtained from solving the PY closure are surprisingly simple (polynomial) expressions. Considerable insight into the structure of HS fluids was gained by introducing a new geometrical interpretation of the direct correlation functions [40]. This insight eventually led to the development of the FMT density functional for HS. In the present work the opposite route is followed. Here, starting from the functional we developed in section 2 , the $c_{i j}^{(2)}(r)$ are obtained as second functional derivatives with respect to the density fields. This yields an (approximate) geometrical representation of the pair direct correlation functions of the AO model. Explicit analytic expressions are given below. It is important to note from the outset that the $c_{i j}^{(2)}(r)$ derived from the present DFT are not (except for special cases) equivalent to those obtained by solving the PY closure for the AO model. In particular, the core condition on the pair correlation functions, $g_{i j}\left(r<R_{i}+R_{j}\right)=0$, is violated in the present approximation, if pair and direct correlation functions are related via the $\mathrm{OZ}$ relation. Nevertheless, in the light of the discussion of section 2.6, we do expect $c_{i j}^{(2)}$ to be similar to those from PY. Recall that the latter can only be obtained numerically for the AO model. The direct correlation functions are given by

$$
c_{i j}^{(2)}\left(\boldsymbol{r}, \boldsymbol{r}^{\prime}\right)=-\frac{\delta^{2} \beta F_{\mathrm{exc}}}{\delta \rho_{i}(\boldsymbol{r}) \delta \rho_{j}\left(\boldsymbol{r}^{\prime}\right)}=-\sum_{\nu, \gamma} \psi_{\nu \gamma}^{i j} w_{\nu}^{i} \otimes w_{\gamma}^{j},
$$

where the $\otimes$ denotes the convolution and $\psi_{\nu \gamma}^{i j}=\partial^{2} \Phi /\left(\partial n_{\nu}^{i} \partial n_{\gamma}^{i}\right)$, and as usual $i, j=\mathrm{c}, \mathrm{p} . \Phi$ is the free-energy density given by equations (24)-(31). Carrying out this analysis reveals that the bulk direct correlation functions possess the following structure:

$$
\begin{aligned}
& c_{\mathrm{cc}}^{(2)}\left(\eta_{\mathrm{c}}, \eta_{\mathrm{p}} ; r\right)=c_{\mathrm{hs}}^{(2)}\left(\eta_{\mathrm{c}} ; r\right)+\eta_{\mathrm{p}} c_{*}^{(2)}\left(\eta_{\mathrm{c}} ; r\right), \\
& c_{\mathrm{cp}}^{(2)}\left(\eta_{\mathrm{c}}, \eta_{\mathrm{p}} ; r\right)=c_{\mathrm{cp}}^{(2)}\left(\eta_{\mathrm{c}} ; r\right), \\
& c_{\mathrm{pp}}^{(2)}\left(\eta_{\mathrm{c}}, \eta_{\mathrm{p}} ; r\right)=0,
\end{aligned}
$$

where $c_{\mathrm{hs}}^{(2)}$ is the solution of the PY closure for one-component HS. The simple dependence on $\eta_{\mathrm{p}}$ originates from the linearity of $F_{\mathrm{exc}}$ in the polymer density profile. This dependence allows $c_{\mathrm{cc}}^{(2)}$ to be split into two parts: $c_{\mathrm{hs}}^{(2)}$ is the residual contribution, present even if polymers are absent, while $c_{*}^{(2)}$ is the contribution from introducing the polymers, i.e. the function $c_{*}^{(2)}$ 
describes the part of the direct correlations between pairs of colloid due to the presence of the polymers. The spatial dependence of $c_{*}^{(2)}$ is similar to that of $c_{\mathrm{hs}}^{(2)}$, and will be discussed below. Linearity of the functional in $\rho_{\mathrm{p}}(\boldsymbol{r})$ implies that $c_{\mathrm{cp}}^{(2)}$ is independent of the polymer density. The size ratio $q$ determines the shape of $c_{\mathrm{cp}}^{(2)}$. Finally, the linearity ensures that the polymer-polymer direct correlation function vanishes, as in the PY closure for the AO model. These observations are, of course, consistent with the low density expansion of $c_{i j}^{(2)}$ used in the alternative derivation of the functional given in section 2.6.

The range of the $c_{i j}^{(2)}$ is determined by the geometric nature of the weight functions $w_{v}^{i}$. As these are characteristic of single-particle geometries, it is clear that the $c_{i j}^{(2)}$ must vanish beyond a separation which is the sum of both particle radii involved, i.e. $c_{i j}^{(2)}\left(r>R_{i}+R_{j}\right)=0$, as is found in the PY treatment. Clearly, this property reflects the approximate nature of the functional. In an exact treatment we would expect contributions beyond the range $R_{i}+R_{j}$.

We now give explicit expressions for $c_{i j}^{(2)}$ entering equations (75)-(77). Recall that the PY solution [41] for HS with packing fraction $\eta_{\mathrm{c}}$ and diameter $\sigma_{\mathrm{c}}\left(\equiv 2 R_{\mathrm{c}}\right)$ is

$$
c_{\mathrm{hs}}^{(2)}\left(\eta_{\mathrm{c}} ; x\right)=-\frac{\Theta\left(\sigma_{\mathrm{c}}-r\right)}{\left(1-\eta_{\mathrm{c}}\right)^{4}} \sum_{i} \tau_{i}^{\mathrm{hs}} x^{i},
$$

where $\tau_{i}^{\mathrm{hs}}$ are functions of the colloid packing fraction $\eta_{\mathrm{c}}$ and $x=r / \sigma_{\mathrm{c}}$. The only non-vanishing coefficients are

$$
\begin{aligned}
\tau_{0}^{\mathrm{hs}} & =\left(1+2 \eta_{\mathrm{c}}\right)^{2}, \\
\tau_{1}^{\mathrm{hs}} & =-\frac{3 \eta_{\mathrm{c}}}{2}\left(2+\eta_{\mathrm{c}}\right)^{2}, \\
\tau_{3}^{\mathrm{hs}} & =\frac{\eta_{\mathrm{c}}}{2}\left(1+2 \eta_{\mathrm{c}}\right)^{2} .
\end{aligned}
$$

The polymer contribution to $c_{\mathrm{cc}}^{(2)}$ is given by

$$
c_{*}^{(2)}\left(\eta_{\mathrm{c}} ; r\right)=-\frac{\Theta\left(\sigma_{\mathrm{c}}-r\right)}{2 q^{3}} \sum_{i} \tau_{i}^{*} x^{i},
$$

where the only non-vanishing coefficients are

$$
\begin{aligned}
& \tau_{0}^{*}=2\left[1+A+2 A \gamma+4 B \gamma+6 B \gamma^{2}+3 C \gamma^{2}(3+4 \gamma)\right], \\
& \tau_{1}^{*}=-3-2 A+(2 B) / 3-6 A \gamma-8 B \gamma-18 B \gamma^{2}-2 C \gamma[-1+9 \gamma(1+2 \gamma)], \\
& \tau_{3}^{*}=1+2 A \gamma+6 B \gamma^{2}+12 C \gamma^{3},
\end{aligned}
$$

where $A, B, C$ and $\gamma$ are defined below equation (69). The limiting form at small colloid density is

$$
c_{*}^{(2)}\left(\eta_{\mathrm{c}} \rightarrow 0 ; r\right)= \begin{cases}-2(1+q)^{3}+3(1+q)^{2} x-x^{3}, & r<\sigma_{\mathrm{c}}, \\ 0, & \text { otherwise. }\end{cases}
$$

The colloid-polymer direct correlation function is given by

$$
c_{\mathrm{cp}}^{(2)}\left(\eta_{\mathrm{c}}, r\right)= \begin{cases}-(1+\gamma)\left(1+A \gamma+2 B \gamma^{2}+3 C \gamma^{3}\right), & r<\left|R_{\mathrm{c}}-R_{\mathrm{p}}\right| \\ -(1+\gamma) \sum_{i} \tau_{\mathrm{cp}, i} x^{i}, & \left|R_{\mathrm{c}}-R_{\mathrm{p}}\right| \leqslant r \leqslant\left|R_{\mathrm{c}}+R_{\mathrm{p}}\right| \\ 0, & \text { otherwise, }\end{cases}
$$


where the only non-vanishing coefficients are

$$
\begin{aligned}
& \tau_{\mathrm{cp},-1}=-\frac{3 \gamma}{32}(1-q)^{2}[3+q+3 \gamma(1+q)]^{2}, \\
& \tau_{\mathrm{cp}, 0}=\frac{1}{2}\left\{2+\gamma\left[7+3 \gamma(5+3 \gamma)+9 \gamma q^{2}+(1+3 \gamma)^{2} q^{3}+3 q(1+q)\right]\right\}, \\
& \tau_{\mathrm{cp}, 1}=-\frac{3 \gamma}{4}\left[5+3 \gamma(4+3 \gamma)+2 q+6 \gamma q+(q+3 \gamma q)^{2}\right], \\
& \tau_{\mathrm{cp}, 3}=\frac{\gamma}{2}(1+3 \gamma)^{2} .
\end{aligned}
$$

In the limit $\eta_{\mathrm{c}} \rightarrow 0$ the colloid-polymer direct correlation function reduces to the colloidpolymer Mayer function $f_{\mathrm{cp}}(r)$, i.e.

$$
c_{\mathrm{cp}}^{(2)}\left(\eta_{\mathrm{c}} \rightarrow 0 ; r\right)= \begin{cases}-1, & r<R_{\mathrm{c}}+R_{\mathrm{p}} \\ 0, & \text { otherwise. }\end{cases}
$$

Simpler expressions are obtained in the special case $q=1$, where only a single length scale is present, and one can use $c_{*}^{(2)}=\frac{\partial}{\partial \eta_{\mathrm{c}}} c_{\mathrm{hs}}^{(2)}\left(\eta_{\mathrm{c}} ; r\right)$ and $c_{\mathrm{cp}}^{(2)}=c_{\mathrm{hs}}^{(2)}\left(\eta_{\mathrm{c}} ; r\right)$. It follows that

$$
c_{*}^{(2)}\left(\eta_{\mathrm{c}} ; r\right)=-\frac{\Theta\left(\sigma_{\mathrm{c}}-r\right)}{\left(1-\eta_{\mathrm{c}}\right)^{5}} \sum_{i} \tau_{i}^{*} x^{i},
$$

where the only non-vanishing coefficients are

$$
\begin{aligned}
& \tau_{0}^{*}=4\left(2+\eta_{\mathrm{c}}\right)\left(1+2 \eta_{\mathrm{c}}\right), \\
& \tau_{1}^{*}=-\frac{3}{2}\left(2+\eta_{\mathrm{c}}\right)\left[2+\eta_{\mathrm{c}}\left(9+\eta_{\mathrm{c}}\right)\right], \\
& \tau_{3}^{*}=\frac{1}{2}\left(1+2 \eta_{\mathrm{c}}\right)\left[1+\eta_{\mathrm{c}}\left(9+2 \eta_{\mathrm{c}}\right)\right] .
\end{aligned}
$$

In order to illustrate the variation with $r$ and $\eta_{\mathrm{c}}$, we plot the various functions, for $q=0.6$, in figure 3. $c_{\mathrm{hs}}^{(2)}(r)$ is shown in figure 3(a) with $\eta_{\mathrm{c}}$ in the range $0-0.4$. This is the contribution to $c_{\mathrm{cc}}^{(2)}$ arising solely from the 'bare' colloids. The polymers generate an (additive) contribution $\eta_{\mathrm{p}} c_{*}^{(2)}(r)$, where the function $c_{*}^{(2)}(r)$, plotted in figure $3(\mathrm{~b})$, has a similar form to $c_{\mathrm{hs}}^{(2)}(r)$. Changing the size ratio $q$ does not dramatically alter the shape; it merely changes the vertical scale of the plot. Finally, figure $3(\mathrm{c})$ shows $c_{\mathrm{cp}}^{(2)}(r)$ for the same values of $\eta_{\mathrm{c}}$. This function is constant for $r<\left|R_{\mathrm{c}}-R_{\mathrm{p}}\right|=0.2 \sigma_{\mathrm{c}}$ and vanishes for $r>\left|R_{\mathrm{c}}+R_{\mathrm{p}}\right|=0.8 \sigma_{\mathrm{c}}$, for the present size ratio. All three functions become more negative in the core region as $\eta_{\mathrm{c}}$ is increased. Note that the polymer density $\eta_{\mathrm{p}}$ only enters $c_{\mathrm{cc}}^{(2)}$ through the linear dependence in equation (75).

Summarizing, we find it remarkable that the direct correlation functions for the AO model, which is characterized by two thermodynamic variables $\eta_{\mathrm{c}}$ and $\eta_{\mathrm{p}}$, can be described by relatively simple analytic expressions and that all relevant information, for a given size ratio $q$, can be condensed into as few as three plots.

\subsection{Structure factors and criticality}

The total pair correlation functions $h_{i j}(r)=g_{i j}(r)-1$, where $g_{i j}(r)$ are pair correlation functions, are related to the direct correlation functions $c_{i j}^{(2)}(r)$ via the mixture OZ relations [41]. These simplify in Fourier space:

$$
\begin{aligned}
& h_{\mathrm{cc}}(k)=\frac{c_{\mathrm{cc}}^{(2)}(k)+\rho_{\mathrm{p}}\left[\left(c_{\mathrm{cp}}^{(2)}(k)\right)^{2}-c_{\mathrm{cc}}^{(2)}(k) c_{\mathrm{pp}}^{(2)}(k)\right]}{D(k)} \\
& h_{\mathrm{pp}}(k)=\frac{c_{\mathrm{pp}}^{(2)}(k)+\rho_{\mathrm{c}}\left[\left(c_{\mathrm{cp}}^{(2)}(k)\right)^{2}-c_{\mathrm{cc}}^{(2)}(k) c_{\mathrm{pp}}^{(2)}(k)\right]}{D(k)} \\
& h_{\mathrm{cp}}(k)=\frac{c_{\mathrm{cp}}^{(2)}(k)}{D(k)}
\end{aligned}
$$



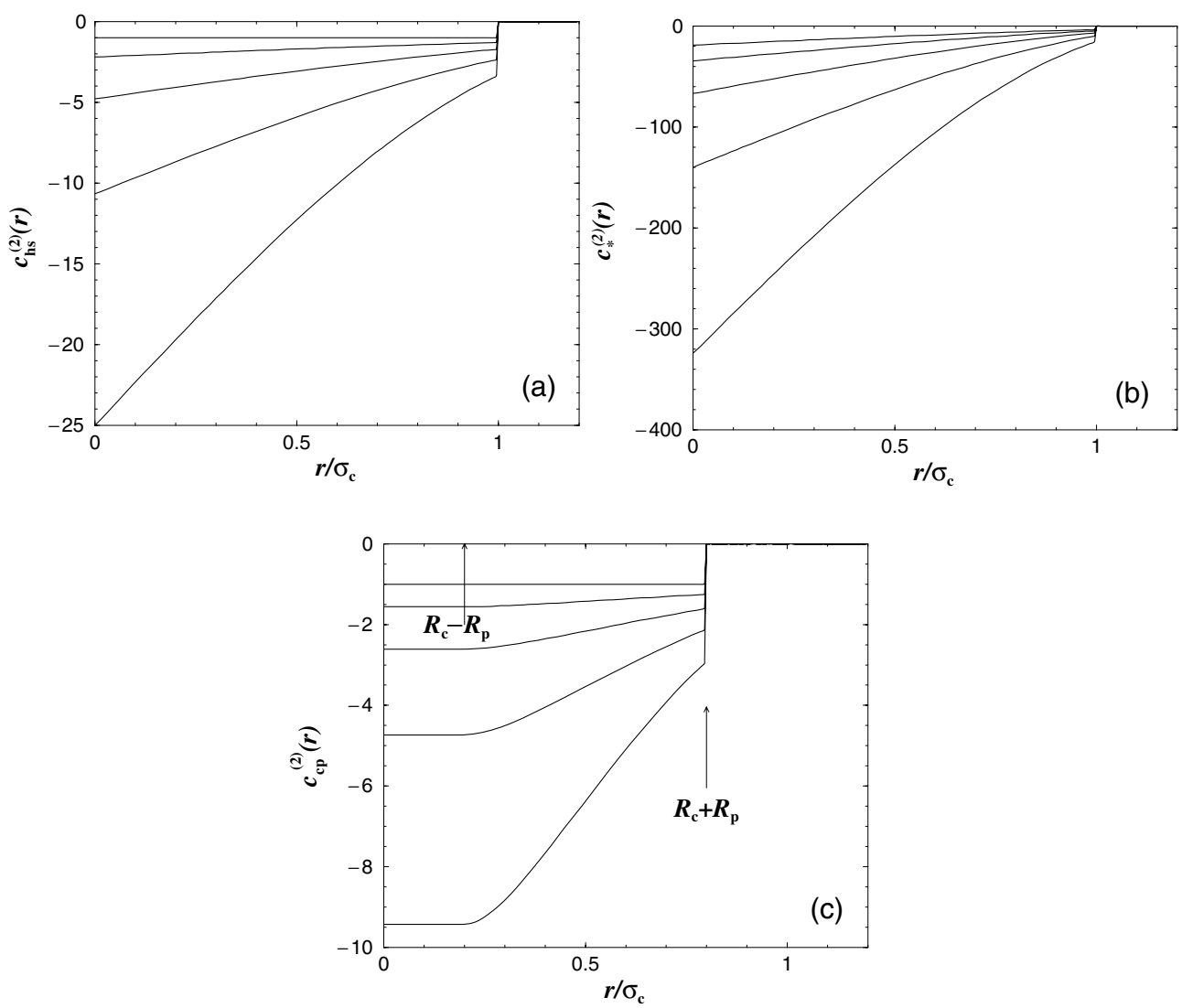

Figure 3. Pair direct correlation functions for $\eta_{\mathrm{c}}=0,0.1,0.2,0.3,0.4$ (from top to bottom) obtained for the AO model with size ratio $q=0.6$ : (a) colloid contribution $c_{\mathrm{hs}}^{(2)}(r)$ to $c_{\mathrm{cc}}^{(2)}(r)$; (b) polymer contribution $c_{*}^{(2)}(r)$ to $c_{\mathrm{cc}}^{(2)}(r)$; (c) full colloid-polymer direct correlation function $c_{\mathrm{cp}}^{(2)}(r)$. These results are obtained from equations (78), (80), (83).

where $h_{i j}(k)$ is the three-dimensional Fourier transform of $h_{i j}(r)$ and the common denominator is given by

$$
D(k)=\left[1-\rho_{\mathrm{c}} c_{\mathrm{cc}}^{(2)}(k)\right]\left[1-\rho_{\mathrm{p}} c_{\mathrm{pp}}^{(2)}(k)\right]-\rho_{\mathrm{c}} \rho_{\mathrm{p}}\left[c_{\mathrm{cp}}^{(2)}(k)\right]^{2} .
$$

The corresponding partial structure factors $S_{i j}(k)$ then can be obtained via [41]

$$
S_{i j}(k)=\delta_{i j}+\left(\rho_{i} \rho_{j}\right)^{1 / 2} h_{i j}(k) .
$$

It is sometimes useful to describe the like-like structure factors by means of the OZ relation

$$
S_{i i}(k)=1 /\left(1-\rho_{i} c_{i i}^{(2), e f f}(k)\right),
$$

that has the same formal structure as the $\mathrm{OZ}$ relation for a one-component system, and that employs an effective direct correlation function given by

$$
c_{i i}^{(2), \text { eff }}(k)=c_{i i}^{(2)}(k)+\frac{\rho_{j} c_{i j}^{(2)}(k)^{2}}{1-\rho_{j} c_{j j}^{(2)}(k)} .
$$

It is straightforward to show that equations (93) and (94) are completely equivalent to the standard mixture formulation of equations (88)-(92). So far all is general but now we note 
that in the present approximation $c_{\mathrm{pp}}^{(2)}=0$, see equation (77). This leads to rather simple expressions for the effective direct correlation functions:

$$
\begin{aligned}
& c_{\mathrm{cc}}^{(2), \text { eff }}(k)=c_{\mathrm{cc}}^{(2)}(k)+\rho_{\mathrm{p}} c_{\mathrm{cp}}^{(2)}(k)^{2}, \\
& c_{\mathrm{pp}}^{(2), \mathrm{eff}}(k)=\frac{\rho_{\mathrm{c}} c_{\mathrm{cp}}^{(2)}(k)^{2}}{\left.1-\rho_{\mathrm{c}} c_{\mathrm{cc}}^{(2)}(k)\right)} .
\end{aligned}
$$

In particular, equation (96) with (93) allows us to understand why $S_{\mathrm{pp}}$ can be significantly different from unity, the ideal gas result, even though $c_{\mathrm{pp}}^{(2)}=0$. Results for $S_{i j}(k)$ and $g_{i j}(r)$ were presented in an earlier letter [31]. We found that for $q=0.15, \eta_{\mathrm{c}}=0.3$ and $\eta_{\mathrm{p}}=0.05$ all three partial pair correlation functions were close to the corresponding PY results. The latter were obtained numerically [8]. For $q=0.1, \eta_{\mathrm{c}}=0.25, \eta_{\mathrm{p}}=0.107$ we compared our results for $S_{\mathrm{cc}}(k)$ and $g_{\mathrm{cc}}(r)$ with those of simulation (for this small size ratio there is an exact mapping to an effective one-component colloid system for which simulations are easily performed [5]). The overall agreement was reasonable, although the structure factor was a little out of phase and the results of the DFT strongly underestimated the very high contact value $g_{\mathrm{cc}}\left(\sigma_{\mathrm{c}}\right)$ and violated (weakly) the core condition $g_{\mathrm{cc}}(r)=0, r<\sigma_{\mathrm{c}}$.

In the present study, we are concerned with less extreme size ratios where the effective (depletion) potential between colloids is longer ranged and less deep near contact than the cases investigated earlier and we expect the DFT to fare somewhat better. An important advantage of the present DFT approach (over most integral equation theories) is that the thermodynamic and structural routes to the spinodal, binodal and, therefore, the critical point are consistent. In particular, the spinodal line, equation (73), can equally well be found by considering the locus of divergence of $S_{i j}(k)$, i.e. the vanishing of $D(k)$ in equation (91). This consistency is especially important in the vicinity of the critical point.

The location of the critical point in the $\eta_{\mathrm{c}}, \eta_{\mathrm{p}, \mathrm{r}}$ plane can be obtained by tracing the coexistence densities towards small $\eta_{\mathrm{p}, \mathrm{r}}$, but careful numerical work is needed to obtain accurate results. In the present case, the critical point can be obtained in a simpler way: starting from the spinodal in the system representation, equation (73), we switch to the reservoir representation using equations (72), (69), and obtain the spinodal polymer reservoir density as a function of colloid density, $\eta_{\mathrm{p}, \mathrm{r}}^{\mathrm{spin}}\left(\eta_{\mathrm{c}}\right)$. As the critical point is at the minimum of this function, finding the minimum is a stable and numerically trivial operation. For states slightly removed from criticality we expect OZ behaviour: $S_{i j}(k)=S_{i j}(0) /\left[1+\xi^{2} k^{2}+\mathrm{O}\left(k^{4}\right)\right]$, where the correlation length $\xi$ is the same for all pairs $i j$. This general result is a direct consequence of the OZ relations for a mixture (88)-(90) and follows from the fact that $D(k)$ is the common denominator for all pairs, equation (91). As the $S_{i j}(k)$ are given analytically in the present theory, we can confirm explicitly the OZ behaviour. We find that the common correlation length diverges with the mean-field exponent $v=1 / 2$ and on a path at fixed $\eta_{\mathrm{c}}=\eta_{\mathrm{c}}^{\text {crit }}$ we define the amplitude $\xi_{0}$ via $\xi=\xi_{0} /\left(\eta_{\mathrm{p}}^{\text {crit }}-\eta_{\mathrm{p}}\right)^{1 / 2}$. $\xi_{0} / \sigma_{\mathrm{c}}$ depends only on the size ratio $q$. It is roughly proportional to the mean of the colloid and polymer diameters and is conveniently expressed as $\xi_{0}=\frac{1}{2}\left(\sigma_{\mathrm{c}}+\sigma_{\mathrm{p}}\right) / K(q)$, where typical values are $K(q)=3.00,2.36, \sqrt{5}$, for $q=0.4,0.8, \infty$, respectively. Note that $\sigma_{\mathrm{p}}=2 R_{\mathrm{p}}$. Figure 4 displays the partial structure factors calculated for $q=0.6$ and a fixed value of $\eta_{\mathrm{c}}$, equal to the critical point value $\eta_{\mathrm{c}}^{\text {crit }}=0.1843$. Results are presented for four values of $\eta_{\mathrm{p}, \mathrm{r}}$, corresponding to the critical 'isochore' in figure 1(a). For $\eta_{\mathrm{p}, \mathrm{r}}=0$ (no polymer) $S_{\mathrm{cc}}(k)$ is simply the (PY) HS structure factor for $\eta_{\mathrm{c}}=0.1843$. Upon increasing $\eta_{\mathrm{p}, \mathrm{r}}, S_{\mathrm{cc}}(k)$ develops a maximum at $k=0$, OZ behaviour ensues and then, at the critical value $\eta_{\mathrm{p}, \mathrm{r}}^{\text {crit }}=0.4943, S_{\mathrm{cc}}(0) \rightarrow+\infty$. $S_{\mathrm{pp}}(k)$ has a somewhat different variation. This function develops its leading maximum at $k=0$ for very small polymer fractions and $S_{\mathrm{pp}}(0)$ is very large even for $\eta_{\mathrm{p}, \mathrm{r}}=0.36$, well away from the critical point. As $\eta_{\mathrm{p}, \mathrm{r}} \rightarrow \eta_{\mathrm{p}, \mathrm{r}}^{\text {crit }}, S_{\mathrm{pp}}(0)$ 


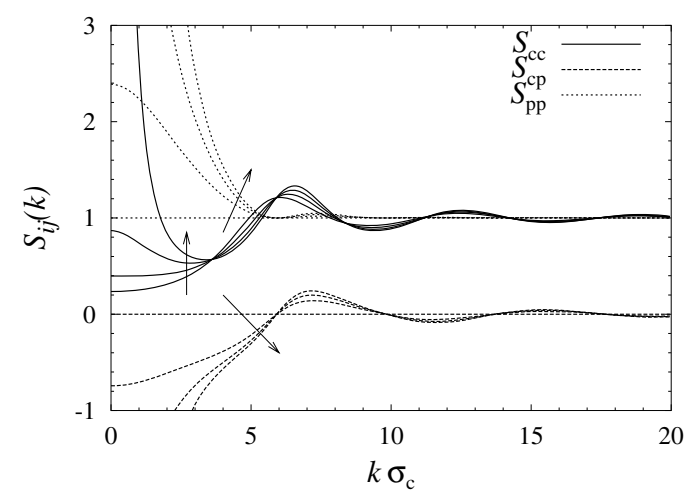

Figure 4. Partial structure factors $S_{\mathrm{cc}}, S_{\mathrm{cp}}, S_{\mathrm{pp}}$ for size ratio $q=0.6$ at fixed colloid fraction $\eta_{\mathrm{c}}=\eta_{\mathrm{c}}^{\text {crit }}=0.1843$, and increasing (indicated by arrows) polymer reservoir density $\eta_{\mathrm{p}, \mathrm{r}}=$ $0,0.2,0.36, \eta_{\mathrm{p}, \mathrm{r}}^{\text {crit }}=0.4943$. On approaching the critical point, along a vertical path in the phase diagram of figure $1(\mathrm{a}), S_{\mathrm{cc}}(k)$ and $S_{\mathrm{pp}}(k)$ become increasingly positive and $S_{\mathrm{cp}}(k)$ increasingly negative at small wavenumbers $k$. Note that for $\eta_{\mathrm{p}, \mathrm{r}}=0, S_{\mathrm{cc}}(k)$ corresponds to the pure HS structure factor.

diverges in the same fashion as $S_{\mathrm{cc}}(0)$. By contrast, $S_{\mathrm{cp}}(0)$ becomes increasingly negative and diverges to $-\infty$ on approaching the critical point. Similar features are found for other size ratios.

The form of $S_{\mathrm{pp}}(k)$ is particularly striking and has repercussions for the distribution of polymer in the mixture. Note that similar shapes of $S_{\mathrm{pp}}(k)$ were found in PY calculations [7,8] for the $\mathrm{AO}$ mixture.

\subsection{Asymptotic decay of correlations: Fisher-Widom line}

The pair correlation functions $g_{i j}(r)$ should exhibit qualitatively different asymptotic decay at different points in the phase diagram. In the neighbourhood of the critical point $g_{i j}(r)$ will decay monotonically, as befits $\mathrm{OZ}$ behaviour, whereas for small values of $\eta_{\mathrm{p}, \mathrm{r}}$ the mixture is HS-like and the $g_{i j}(r)$ should exhibit damped oscillatory decay as $r \rightarrow \infty$. Thus upon varying the thermodynamic parameters $\eta_{\mathrm{p}, \mathrm{r}}$ and $\eta_{\mathrm{c}}$ the ultimate decay of $g_{i j}(r)$ should change from being oscillatory to purely monotonic [42-45]. The line in the phase diagram separating the two types of decay is termed the FW line [42] after the authors who introduced the concept. This is not a line of thermodynamic singularity. Rather it indicates crossover to different structural regimes. Although the FW line is defined by considering the decay of bulk pair correlation functions, it plays a key role in inhomogeneous situations since the asymptotic decay into bulk of one-body density profiles is determined by the same physical considerations. Thus it is relevant for several interfacial phenomena [32, 43, 44, 46].

In order to calculate the FW line, we again exploit the fact that our partial structure factors are given analytically. Since the asymptotic decay of $g_{i j}(r)$ is determined by the singularities of the $S_{i j}(k)$ in the complex plane our strategy is to trace the locations of these. We denote these singularities $k=a_{1}+\mathrm{i} a_{0}$. Oscillatory behaviour, $h_{i j}(r \rightarrow \infty) \propto \cos \left(a_{1} r\right) \exp \left(-a_{0} r\right) / r$, stems from poles with non-zero real part $a_{1}$, whereas monotonic behaviour, $h(r \rightarrow \infty) \propto$ $\exp \left(-a_{0} r\right) / r$, stems from poles residing on the imaginary axis, $a_{1}=0$. The ultimate decay is governed by the pole with the smallest imaginary part $a_{0}$ [44]. We obtain the location of the singularities by finding the roots of $1 /\left|S_{i j}(k)\right|=0$ numerically, taking appropriate starting values. This is equivalent to finding the zeros of $D(k)$, equation (91), in the complex plane. 

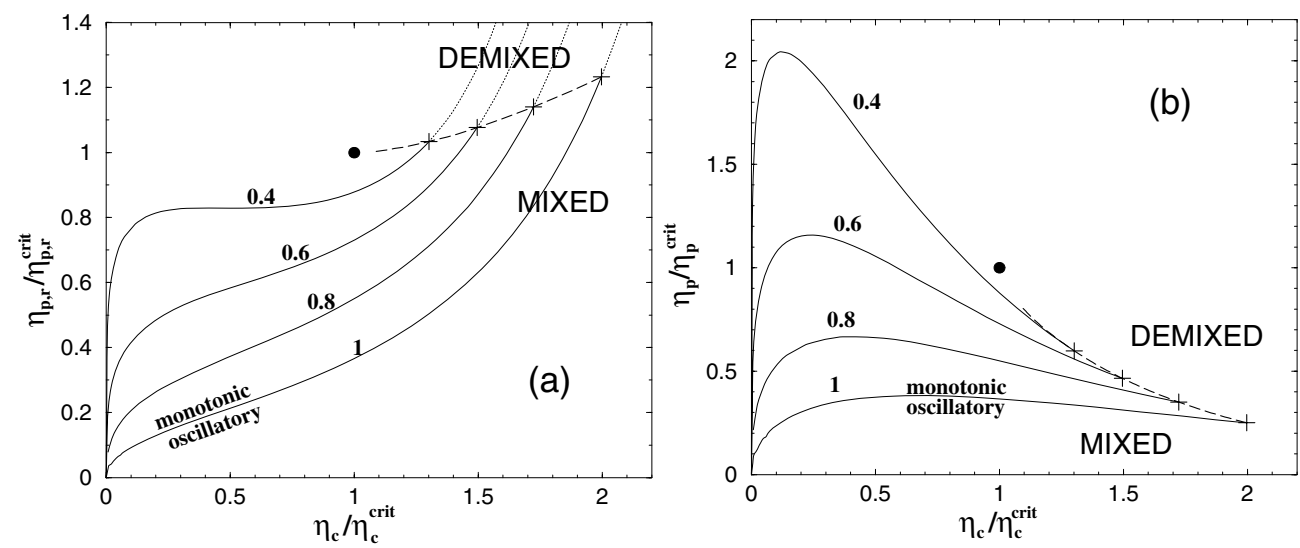

Figure 5. FW lines for size ratios $q=0.4,0.6,0.8,1$ plotted in scaled variables. The FW lines are denoted by solid curves; the dotted extensions are metastable, i.e. they lie inside the binodal. The intersections of the FW lines and the binodal are crosses joined by a dashed curve. (a) Scaled reservoir representation with $\eta_{\mathrm{c}} / \eta_{\mathrm{c}}^{\text {crit }}$ and $\eta_{\mathrm{p}, \mathrm{r}} / \eta_{\mathrm{p}, \mathrm{r}}^{\text {crit }}$; (b) scaled system representation with $\eta_{\mathrm{c}} / \eta_{\mathrm{c}}^{\text {crit }}$ and $\eta_{\mathrm{p}} / \eta_{\mathrm{p}}^{\text {crit }}$. For each $q$, increasing $\eta_{\mathrm{p}, \mathrm{r}}$ or $\eta_{\mathrm{p}}$ at fixed $\eta_{\mathrm{c}}$ leads to crossover from monotonic to oscillatory decay at the FW line.

Note that all partial structure factors possess the same singularities, because of the common denominator $D(k)$. That is why all the $h_{i j}(r)$ exhibit the same exponential decay length $a_{0}^{-1}$ and, when oscillatory, the same wavelength $2 \pi / a_{1}$, and why there is a single FW line that characterizes the crossover in a mixture. In practice we chose $S_{\mathrm{cc}}(k)$ and calculated the pole with the smallest $a_{0}$ residing on the imaginary axis and also the pole with the smallest $a_{0}$ but with $a_{1}>0$. At fixed $\eta_{\mathrm{c}}$, we varied $\eta_{\mathrm{p}}$ until the imaginary parts $a_{0}$ of both poles were identical, giving a point on the FW line. Following this procedure for all $\eta_{\mathrm{c}}$ maps out the complete FW line for a given size ratio. In figure 1 we plot the FW line for $q=0.6$ in both the reservoir and system representations. The FW line intersects the binodal on the colloid-rich side (liquid) and is bounded at high $\eta_{\mathrm{p}, \mathrm{r}}$ by the liquid spinodal. For this size ratio the overall shape and location of the FW line appears similar to that found $[43,45]$ for simple fluids, whose interatomic pair potential is of finite range and which exhibit liquid-gas phase separation, once we recall that $\eta_{\mathrm{p}, \mathrm{r}}$ is equivalent to inverse temperature. Figure 2 displays the intersection of the FW line with the binodal for several values of size ratio $q$. As $q$ increases the intersection occurs further from the critical point, exposing a larger range of monotonic decay along the liquid side of the binodal. In figure 5 we plot the FW lines in terms of the scaled variables $\eta_{\mathrm{c}} / \eta_{\mathrm{c}}^{\text {crit }}$ and $\eta_{\mathrm{p}, \mathrm{r}} / \eta_{\mathrm{p}, \mathrm{r}}^{\text {crit }}$ (or $\eta_{\mathrm{p}} / \eta_{\mathrm{p}}^{\text {crit }}$ ) for four values of $q$. As $q$ increases we observe that a larger portion of the scaled phase diagram lies on the monotonic side of the FW line. For $q=0.4$ the FW line lies just below the critical point and most of the single-phase portion of the scaled phase diagram now falls on the oscillatory side. This might reflect the fact that for $q=0.4$ (and smaller values of $q$ ) the FW line exhibits a minimum when plotted in the reservoir representation, figure 5(a). The presence of the minimum implies that on increasing $\eta_{\mathrm{c}}$ at an appropriate fixed $\eta_{\mathrm{p}, \mathrm{r}}$ the decay of correlation functions should change from monotonic to oscillatory to monotonic and, finally, to oscillatory. Whether such behaviour reflects the fact that the fluid-fluid phase separation should be close to becoming metastable (w.r.t. fluid-solid) for these small values of $q$ can only be ascertained by more sophisticated treatments. What is probably more relevant for a real colloid-polymer mixture is figure 5(b) which shows that in the system representation there is a maximum in the FW line for all four values of $q$. Thus increasing $\eta_{\mathrm{c}}$ at a sufficiently 

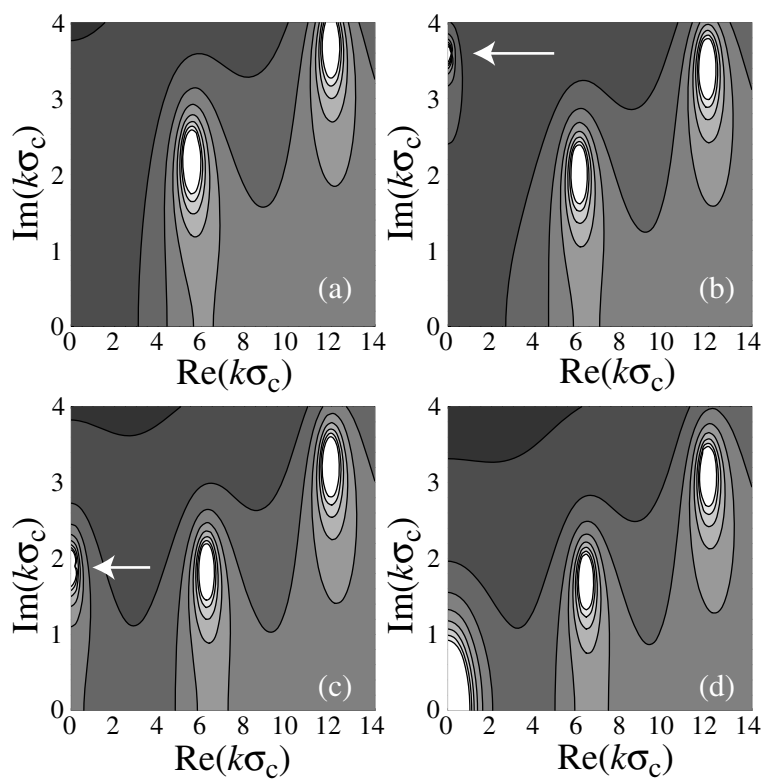

Figure 6. Absolute value of the colloid-colloid structure factor $\left|S_{\mathrm{cc}}\right|$ in the complex plane for size ratio $q=0.6$ at fixed colloid fraction $\eta_{\mathrm{c}}=\eta_{\mathrm{c}}^{\text {crit }}=0.1843$, and four values of polymer reservoir density $\eta_{\mathrm{p}, \mathrm{r}}$ : (a) pure HS, $\eta_{\mathrm{p}, \mathrm{r}}=0\left(\eta_{\mathrm{p}}=0\right)$; (b) $\eta_{\mathrm{p}, \mathrm{r}}=0.2\left(\eta_{\mathrm{p}}=0.07161\right)$ in the oscillatory regime; (c) on the FW line, $\eta_{\mathrm{p}, \mathrm{r}}=0.36\left(\eta_{\mathrm{p}}=0.1290\right)$; (d) at the critical point, $\eta_{\mathrm{p}, \mathrm{r}}=0.4943$ $\left(\eta_{\mathrm{p}}=0.1770\right)$. Arrows indicate the (smallest) purely imaginary pole. This pole lies at the origin in (d). Bright (dark) colour indicates large (small) values. Note that these statepoints are the same as in figure 4.

small fixed polymer fraction $\eta_{\mathrm{p}}$ should lead to crossover from monotonic to oscillatory back to monotonic decay of correlations (see also figure 1(b)). Finally, we remark that in the limit $\eta_{\mathrm{p}, \mathrm{r}} \rightarrow 0$, the FW line smoothly approaches the origin, $\eta_{\mathrm{c}}=0$. This is to be expected, since for pure HS $g(r)$ exhibits oscillatory decay for all non-zero values of the packing fraction. Such behaviour is equivalent to that found for simple fluids in the limit $T \rightarrow \infty$ [45]. We can summarize and visualize much of what is described in this subsection by analysing the behaviour of the structure factors in the complex plane, and in figure 6 we report results for $\left|S_{\mathrm{cc}}(k)\right|$ with $q=0.6$, taken along the same critical 'isochore', $\eta_{\mathrm{c}}=\eta_{\mathrm{c}}^{\text {crit }}$, as in figure 4 .

Pure HS (figure 6(a)) do not possess a pole on the imaginary axis so the two maxima correspond to complex poles; the dominant one corresponds to $a_{1} \sigma_{\mathrm{c}} \sim 2 \pi$. Upon increasing the polymer reservoir density to $\eta_{\mathrm{p}, \mathrm{r}}=0.2$ (figure $6(\mathrm{~b})$ ), a pole appears on the imaginary axis and this moves downwards with $\eta_{\mathrm{p}, \mathrm{r}}$, until its value equals that of the imaginary part of the neighbouring complex pole (figure 6(c)) defining a point on the FW line. At the critical point, the pole on the imaginary axis reaches the origin (figure $6(d)$ ). This pole leads to the divergence of the physical structure factors, $S_{i j}(k \rightarrow 0)$, at the critical point. Figure 4 displays the physical structure factors at the same statepoints.

\subsection{Effective interaction between colloids: depletion potential}

In their original study Asakura and Oosawa [1] determined the effective potential, $V_{\mathrm{AO}}\left(r ; z_{\mathrm{p}}\right)$, between two HS colloids (or macroparticles) in a sea of ideal polymer of fugacity $z_{\mathrm{p}}$. Their celebrated potential is attractive in the range $2 R_{\mathrm{c}}<r<2\left(R_{\mathrm{c}}+R_{\mathrm{p}}\right)$ and vanishes for larger 
separations $r$. The attraction arises from the depletion effect whereby polymer is excluded or depleted from the region between the colloids when the separation of their surfaces is $<2 R_{\mathrm{p}}$. More formally, $V_{\mathrm{AO}}$ describes the two-body contribution to the effective one-component Hamiltonian, $H^{\text {eff }}$, obtained by integrating out the polymer degrees of freedom in the binary AO mixture. For a homogeneous system $H^{\text {eff }}$ is given by [5]

$\beta H^{\mathrm{eff}}\left(\left\{\boldsymbol{R}_{i}\right\} ; z_{\mathrm{p}}\right)=V z_{\mathrm{p}}\left(-1+\eta_{\mathrm{c}}(1+q)^{3}\right)+\beta \sum_{i<j} V_{\mathrm{AO}}\left(\left|\boldsymbol{R}_{i}-\boldsymbol{R}_{j}\right| ; z_{\mathrm{p}}\right)+$ higher body terms,

where the first term is independent of the colloid coordinates $\left\{\boldsymbol{R}_{i}\right\}$. The pair potential is

$\beta V_{\mathrm{AO}}\left(\left\{\boldsymbol{R}_{i}\right\} ; z_{\mathrm{p}}\right)=\beta V_{\mathrm{cc}}\left(\left|\boldsymbol{R}_{i}-\boldsymbol{R}_{j}\right|\right)-z_{\mathrm{p}} \int \mathrm{d}^{3} r f_{\mathrm{cp}}\left(\boldsymbol{R}_{i}-\boldsymbol{r}\right) f_{\mathrm{cp}}\left(\boldsymbol{R}_{j}-\boldsymbol{r}\right)$,

where $V_{\mathrm{cc}}$ is the direct colloid-colloid (HS) interaction and $f_{\mathrm{cp}}$ is the colloid-polymer Mayer function. The second term in equation (98) is called the depletion potential. Another interpretation of $V_{\mathrm{AO}}$ comes from considering the pair correlation functions $g_{i j}(r)$ in a dilute (in colloid) binary AO mixture. In the limit $\eta_{\mathrm{c}} \rightarrow 0, g_{\mathrm{cc}}(r) \approx \exp \left(-\beta V_{\mathrm{AO}}\left(r ; z_{\mathrm{p}}\right)\right)$.

Clearly, any exact treatment of the mixture should yield this exact result. Note that this is equivalent to requiring the effective colloid-colloid direct correlation function, see equation (94), to reduce to

$$
c_{\mathrm{cc}}^{(2), \mathrm{eff}}(r) \approx \exp \left(-\beta V_{\mathrm{AO}}\left(r ; z_{\mathrm{p}}\right)\right)-1
$$

for $\eta_{\mathrm{c}} \rightarrow 0$. It is well known that the PY closure for the AO mixture (and, indeed, for highly asymmetric HS mixtures where $V_{\mathrm{AO}}$ should be replaced by the HS depletion potential) does not yield the correct limiting behaviour. Rather one finds that for low colloid fractions $g_{\mathrm{cc}}(r) \sim 1-\beta V_{\mathrm{AO}}\left(r ; z_{\mathrm{p}}\right)$ for $r>2 R_{\mathrm{c}}$, which implies the PY results for the contact value $g_{\mathrm{cc}}\left(2 R_{\mathrm{c}}^{+}\right)$are too small $[8,47,48]$. Given that our present DFT bears a close resemblance to the PY approximation we might expect to observe similar failings [31]. The advantage here is that we have explicit, analytic results for $c_{i j}^{(2)}$ so it is straightforward to analyse the behaviour of correlations in the low $\eta_{\mathrm{c}}$ limit. From equations (75), (78), (80), (82) we can ascertain the limiting behaviour of $c_{\mathrm{cc}}^{(2) \text {,eff }}(r)$ given by the Fourier transform of equation (95). Since $c_{\mathrm{cc}}^{(2)}\left(\eta_{\mathrm{p}} ; r\right)=0$ for $r>2 R_{\mathrm{c}}$ and $c_{\mathrm{cp}}^{(2)}\left(\eta_{\mathrm{c}} \rightarrow 0 ; r\right)=f_{\mathrm{cp}}(r)$ it follows that DFT predicts, for $\eta_{\mathrm{c}} \rightarrow 0$,

$$
c_{\mathrm{cc}}^{(2), \text { eff }}(r)=-\beta V_{\mathrm{AO}}\left(r ; z_{\mathrm{p}}\right), \quad r>2 R_{\mathrm{c}},
$$

where we used the fact that $z_{\mathrm{p}}=\rho_{\mathrm{p}, \mathrm{r}}=\rho_{\mathrm{p}}$ in the limit $\eta_{\mathrm{c}} \rightarrow 0$. Thus our DFT does not yield the exact limiting result (99); the latter only reduces to (100) in the limit $z_{\mathrm{p}} \rightarrow 0$. The present approach, via the OZ route to the pair correlation functions, suffers from the same failing as the PY approximation. Clearly both approximation schemes fail to incorporate the physical ingredients which are required to exponentiate $-\beta V_{\mathrm{AO}}$.

However, we must not infer that the present DFT fails to incorporate the depletion effect which is, after all, the crucial feature of colloid-polymer mixtures. We have already seen in section 3.1 that the bulk free energy of the mixture acquires an attractive contribution; this contribution can only arise from the depletion effect. Moreover, the theory does generate an attractive contribution in $c_{\mathrm{cc}}^{(2), e f f}$, equation (100), albeit one that is too weak.

Within DFT the OZ route is not the only one to pair correlation functions. The alternative is the test particle route, whereby one fixes a particle of species $i$ at the origin and determines the inhomogeneous one-body density profile $\rho_{j}(\boldsymbol{r})$ arising from the external potential $V_{i j}(r)$ exerted by the fixed particle. It follows that $g_{i j}(r)=\rho_{j}(r) / \rho_{j}(\infty)$. In applying the test particle prescription one solves the Euler-Lagrange equations for the one-body densities so all 
that is required are the one-body direct correlation functions $c_{i}^{(1)}(r)$; these involve only a first derivative of $F_{\text {exc }}$, see equation (60). One can show that in the limit $\eta_{\mathrm{c}} \rightarrow 0$, the test particle route for the present DFT yields the exact result $g_{\mathrm{cc}}(r) \approx \exp \left(-\beta V_{\mathrm{AO}}\left(r ; z_{\mathrm{p}}\right)\right)$. An instructive way of obtaining this result is to implement the procedure developed by Roth et al [38] for calculating the depletion potential. The latter is given by

$$
-\beta V_{\mathrm{dep}}\left(r ; z_{\mathrm{p}}\right)=\lim _{z_{\mathrm{c}} \rightarrow 0}\left[c_{\mathrm{c}}^{(1)}(r)-c_{\mathrm{c}}^{(1)}(\infty)\right],
$$

where $c_{\mathrm{c}}^{(1)}(r)$ refers to the situation where a colloid, fixed at the origin, exerts its field on the polymer and on a (test) colloid inserted at $r$ [38]. $c_{\mathrm{c}}^{(1)}(r)$ is easily calculated for the present DFT (the calculation mimics that for the binary HS functional given in [38]) and in the limit where the colloid density vanishes we find that $\beta V_{\text {dep }}$ is proportional to the convolution of two Mayer functions, i.e. it reduces precisely to the second term in equation (98). That the analysis can be performed analytically and the result is much simpler than that for HS mixtures simply reflects the fact that $f_{\mathrm{pp}}(r)=0$ in the AO model.

With hindsight it is, perhaps, not too surprising that geometrically based DFT should describe correctly the depletion potential; the latter is merely a manifestation of excluded volume effects. Nevertheless, this excercise does indicate that the test particle route to $g_{\mathrm{cc}}(r)$ is likely to be much more accurate than the OZ route, especially for situations where $\eta_{\mathrm{c}}$ is low and $\eta_{\mathrm{p}, \mathrm{r}}$ is high so that the depletion potential is strongly attractive and contact values $g_{\mathrm{cc}}\left(2 R_{\mathrm{c}}^{+}\right)$ are very large. The test particle route also has the advantage that it guarantees that $g_{\mathrm{cc}}(r)=0$ for $r<2 R_{\mathrm{c}}$ and $g_{\mathrm{cp}}(r)=0$ for $r<R_{\mathrm{c}}+R_{\mathrm{p}}$.

\section{Discussion}

We have derived a DFT for the binary AO mixture based on geometrical information about the hard and ideal spheres which constitute the AO model. Our FMT approach follows the strategy which was employed successfully for other model systems. Imposing the correct dimensional crossover on the functional, section 2, appears to provide a systematic procedure for deriving approximate DFTs. Whether the procedure can be implemented explicitly depends on the particular model. In the present case, as for penetrable spheres [25], the WidomRowlinson model [26], a needle-sphere model [28], a model amphiphilic mixture [29] and a ternary mixture of colloids, polymers and needles [30], the procedure is tractable and leads to a relatively simple functional. An alternative derivation, section 2.6, which corresponds to linearizing the Rosenfeld functional for a binary HS system w.r.t. the density of one of the species, yielded the identical functional. Multi-component mixtures can also be treated by our procedures. For example, if a three-component mixture of HS of two different sizes plus polymer were required one would take the Rosenfeld HS functional for a ternary mixture of HS and linearize in one of the densities which is then identified as (ideal) polymer. Similarly, if a three-component mixture of HS plus two polymer species were required one would simply linearize the ternary HS functional w.r.t. two densities. The corresponding functionals are again identical to those obtained from application of the recipe outlined in section 2.4 , where the generating zero-dimensional free energy $F_{0 \mathrm{~d}}$ is replaced by that for the corresponding ternary mixture. In fact the free energy for the ternary system is simply equation (14) with colloid (polymer) packing fraction replaced by the sum of packing fractions of colloid (polymer) species. In section 2.7 we described the concept of integrating out degrees of freedom within the context of DFT. The present functional affords a valuable example because the EulerLagrange equations decouple in this case and $c_{\mathrm{p}}^{(1)}(\boldsymbol{r})$ is a functional of the colloid profile alone. It is important to emphasize, however, that the mapping to an effective one-component 
fluid of colloids that is implicit within the approximate DFT treatment is not equivalent to that which is obtained by an explicit and exact integrating out of polymer coordinates in the partition function. Recall that this latter route yields an effective Hamiltonian containing only one- and two-body contributions when $q<0.1547$. This particular size ratio has no special significance within our approximate DFT.

Our present paper focuses on the properties of bulk fluid phases. We found that the bulk free energy of the AO mixture is identical to that derived from the free-volume theory of Lekkerkerker et al [4] and we pointed out some of the connections between the two approaches (see section 3.1), emphasizing that both impose linearity of the free energy in the polymer density. Since the two theories yield the same free energy, they predict identical fluid-fluid phase separation, i.e. the same binodal and spinodal. The latter can be determined analytically from equation (73), which is particularly useful for critical point studies. How accurate is the free-volume theory for the phase separation curve? When we completed our paper there were no systematic studies of the full binary AO model for the range of size ratios $q$ where fluidfluid phase separation occurs. Published studies consider either the effective one-component Hamiltonian (97) keeping only pair potential [5], which is strictly valid for $q<0.1547$, or a lattice polymer version [6]. It is well known that treating highly asymmetric mixtures by brute force simulation is beset by ergodicity problems and slow equilibration when the packing fraction of the smaller species is large. A new study by Dijkstra [47] indicates that for $q=1$ the fluid-fluid binodal does lie rather close to the free-volume result. This also seems to be the case for other values of $q$ [50] and the current indications are that free-volume theory (and, therefore, our present DFT) does provide a reasonable account of the location of the fluid-fluid binodal in the $\eta_{\mathrm{c}}, \eta_{\mathrm{p}, \mathrm{r}}$ plane for those values of $q(>0.35)$ where fluid-fluid phase separation is stable w.r.t. fluid-solid. We have not considered solid phases in the present study. These could be investigated within the framework of the present DFT which would enable us to calculate the full phase diagram. Existing studies [4, 5] based on free-volume ideas employ the expression of Hall [49] for the Helmholtz free-energy density of the HS solid entering equation (71) and the same expression for the free-volume fraction $\alpha\left(\rho_{\mathrm{c}}\right)$ as used for fluid phases. It would be interesting to compare DFT predictions for the (rich) fluid-solid phase equilibria in this model with those from other approximate theories and with simulation. Note that the tensor contribution, which depends on the weight function $\hat{\boldsymbol{w}}_{\mathrm{m} 2}^{i}(\boldsymbol{r})$, entering $\Phi_{3}$ in equation (31) is crucially important for the inhomogeneous solid phase [21, 22] but makes no contribution to the free energy of a homogeneous fluid phase.

An important feature of our present DFT is that pair correlation functions of the bulk fluid can be obtained very easily via the OZ route (sections 3.3 and 3.4). Thus the theory is much easier to implement than the PY integral equation theory for the AO mixture which can only be solved numerically [7,8]. The pair direct correlation functions and the partial structure factors $S_{i j}(k)$ are given analytically, as is the case in the PY approximation for HS mixtures. What is remarkable here is that the AO mixture exhibits pure entropically driven fluid-fluid phase separation so that the $S_{i j}(k)$ display a divergence at a spinodal line which is identical to that obtained from the bulk free energy. This type of consistency is, of course, a direct consequence of the DFT approach. Having analytical results for the $S_{i j}(k)$ also enabled us to obtain directly the FW line (section 3.5), describing the line of crossover from monotonic to oscillatory asymptotic decay of the total pair correlation functions $h_{i j}(r)$. To the best of our knowledge this is the first time the FW line has been investigated in detail for this type of system. One intriguing feature, which warrants further attention, is the nature and location of the FW line as $q$ becomes smaller and the fluid-fluid critical point shifts to lower $\eta_{\mathrm{p}}$ and higher $\eta_{\mathrm{c}}$ (figure 2). For $q=0.4$ the monotonic regime, where $h_{i j}(r)$ exhibit OZ-like decay at large $r$, is restricted to a narrow portion of the phase diagram near the critical point - see 
figure 5. This might be a structural indicator that the fluid-fluid phase separation is becoming metastable w.r.t. fluid-solid.

We expect the accuracy of the $h_{i j}(r)$ and $S_{i j}(k)$ obtained from the OZ route to be comparable with that of the PY approximation for the AO mixture. As remarked in section 3.6, both approximations fail to reproduce the correct limiting behaviour of $g_{\mathrm{cc}}(r)$ as $\eta_{\mathrm{c}} \rightarrow 0$. This is particularly significant for small size ratios at states with low $\eta_{\mathrm{c}}$ and high values of $\eta_{\mathrm{p}, \mathrm{r}}$ where the depletion potential is very deep and very short ranged. Neither PY nor the present theory can be expected to yield accurate pair correlation functions in this regime. The DFT has the additional drawback of not enforcing the core conditions $g_{\mathrm{cc}}\left(r<2 R_{\mathrm{c}}\right)=0$ and $g_{\mathrm{cp}}\left(r<R_{\mathrm{c}}+R_{\mathrm{p}}\right)=0$. These core conditions constitute implicit (hidden) constraints when viewed from the perspective of taking functional derivatives to obtain $c_{i j}^{(2)}(r)$ and then inverting to obtain $g_{i j}(r)$. In principle, one might exploit the core conditions in order to derive (more sophisticated) approximations for the free-energy functional. In practice, such a procedure would be extremely difficult to implement. (In this context it appears remarkable that Rosenfeld's HS functional does yield $g_{i j}(r)$ that fulfill the core conditions. Of course, it is by virtue of the fact that the $c_{i j}^{(2)}$ generated from the Rosenfeld functional are those of PY that the resulting $g_{i j}(r)$ do satisfy the core condition.) DFT provides an alternative route to $g_{i j}(r)$, namely the test-particle procedure, and we have argued that this is likely to yield significantly better results than the OZ route, especially for situations where the depletion attraction is very strong. Once again systematic comparisons with simulation results are required in order to assess further the degree of consistency and, therefore, the reliability of the DFT. Note that the $\mathrm{OZ}$ and test particle routes do yield identical results for the decay length $a_{0}^{-1}$ and wavelength $2 \pi / a_{1}$, characterizing the asymptotic decay of the pair correlation functions. It follows that the FW lines calculated from both routes are identical.

The experience gleaned from the present study of bulk properties gives us confidence in applying our DFT to inhomogeneous fluids where the spatial variation of the density profiles arises from the imposition of external fields. Since the DFT incorporates fluid-fluid phase separation it has been applied $[9,32]$ to

(i) the planar interface between coexisting colloid-rich and colloid-poor phases-the density profiles of both colloid and polymer exhibit oscillations on the colloid-rich (liquid) side of the interface provided the bulk liquid lies on the oscillatory side of the FW line - and

(ii) the study of wetting phenomena for the mixture adsorbed at a hard wall. The interface between the wall and the colloid-poor phase is partially wetted by the colloid-rich phase at high $\eta_{\mathrm{p}, \mathrm{r}}$ but is completely wet at low $\eta_{\mathrm{p}, \mathrm{r}}\left(>\eta_{\mathrm{p}, \mathrm{r}}^{\text {crit }}\right)$.

A novel sequence of layering transitions accompanies the transition to complete wetting. It was argued [32] that the surface phase behaviour reflects the presence of many-body contributions to the effective one-component Hamiltonian for the colloids. Such contributions are included implicitly in the DFT. Other adsorption problems which can be tackled by our DFT include possible wall-induced crystallization (depletion at a hard wall leads to a very high contact density for colloids [13]) and wetting of the wall-fluid interface by the crystalline phase. Mixtures confined by planar walls or in other geometries are readily tackled which means that phenomena such as capillary condensation can be investigated in detail. Sedimentation in a gravitational field, behaviour in laser fields and the properties of solid-fluid interfaces are also topics ripe for investigation. 


\section{Acknowledgments}

We thank M Dijkstra, R van Roij, R Roth, A Lang, P Tarazona, M Fuchs and I Götze for many helpful discussions. This research was supported by the British-German ARC Programme (project104b).

\section{Appendix A. Weight functions in Fourier space}

For completeness we list the (three-dimensional) Fourier transforms of the first six weight functions listed in equations (17)-(22):

$$
\begin{aligned}
& w_{3}^{i}(\boldsymbol{k})=-\frac{4 \pi}{k^{3}}\left[k R_{i} \cos \left(k R_{i}\right)-\sin \left(k R_{i}\right)\right], \\
& w_{2}^{i}(\boldsymbol{k})=\frac{4 \pi R_{i}}{k} \sin \left(k R_{i}\right), \\
& w_{1}^{i}(\boldsymbol{k})=\frac{\sin \left(k R_{i}\right)}{k}, \\
& w_{0}^{i}(\boldsymbol{k})=\frac{\sin \left(k R_{i}\right)}{R_{i} k}, \\
& \boldsymbol{w}_{\mathrm{v} 2}^{i}(\boldsymbol{k})=\frac{4 \pi}{k^{2}}\left[k R_{i} \cos \left(k R_{i}\right)-\sin \left(k R_{i}\right)\right] \frac{\boldsymbol{k}}{k}, \\
& \boldsymbol{w}_{\mathrm{v} 1}^{i}(\boldsymbol{k})=\frac{k R_{i} \cos \left(k R_{i}\right)-\sin \left(k R_{i}\right)}{k^{2} R_{i}},
\end{aligned}
$$

where $k=|k|$.

\section{Appendix B. Density functional in two dimensions}

In the following we present the DF for the two-dimensional AO model obtained from the route described in section 2.6 starting from the two-dimensional Rosenfeld hard disc functional (see e.g. [20]). The excess free energy $F_{\text {exc }}$ has the general structure, equation (15) of a two-dimensional integral over a free-energy density. The weighted densities are obtained by two-dimensional convolutions

$$
n_{v}^{i}(x)=\int \mathrm{d}^{2} r \rho_{i}(\boldsymbol{r}) w_{v}^{i}(\boldsymbol{x}-\boldsymbol{r})
$$

of the four two-dimensional weight functions

$$
\begin{aligned}
& w_{d}^{i}(\boldsymbol{r})=\Theta\left(R_{i}-r\right), \\
& w_{1}^{i}(\boldsymbol{r})=\delta\left(R_{i}-r\right), \\
& \boldsymbol{w}_{\mathrm{v} 1}^{i}(\boldsymbol{r})=\delta\left(R_{i}-r\right) \boldsymbol{r} / r, \\
& w_{0}^{i}(\boldsymbol{r})=\delta\left(R_{i}-r\right) /(2 \pi r),
\end{aligned}
$$

where $r$ in equations (B.2)-(B.5) denotes the two-dimensional position vector. The free-energy density is

$$
\begin{gathered}
\Phi=-\left(n_{0}^{\mathrm{c}}+n_{0}^{\mathrm{p}}\right) \ln \left(1-n_{d}^{\mathrm{c}}\right)+\frac{n_{0}^{\mathrm{c}} n_{d}^{\mathrm{p}}}{1-n_{d}^{\mathrm{c}}}+\frac{n_{1}^{\mathrm{c}} n_{1}^{\mathrm{c}}-\boldsymbol{n}_{\mathrm{v} 1}^{\mathrm{c}} \cdot \boldsymbol{n}_{\mathrm{v} 1}^{\mathrm{c}}}{4 \pi}\left[\frac{1}{1-n_{d}^{\mathrm{c}}}+\frac{n_{d}^{\mathrm{p}}}{\left(1-n_{d}^{\mathrm{c}}\right)^{2}}\right] \\
+\frac{n_{1}^{\mathrm{p}} n_{1}^{\mathrm{c}}-\boldsymbol{n}_{\mathrm{v} 1}^{\mathrm{p}} \cdot \boldsymbol{n}_{\mathrm{v} 1}^{\mathrm{c}}}{2 \pi\left(1-n_{d}^{\mathrm{c}}\right)} .
\end{gathered}
$$




\section{References}

[1] Asakura S and Oosawa F 1954 J. Chem. Phys. 221255

[2] Vrij A 1976 Pure Appl. Chem. 48471

[3] Gast A P, Hall C K and Russell W B 1983 J. Colloid Interface Sci. 96251

[4] Lekkerkerker H N W, Poon W C K, Pusey P N, Stroobants A and Warren P B 1992 Europhys. Lett. 20559

[5] Dijkstra M, Brader J M and Evans R 1999 J. Phys.: Condens. Matter 1110079

[6] Meijer E J and Frenkel D 1994 J. Chem. Phys. 1006873

[7] Louis A A, Finken R and Hansen J 1999 Europhys. Lett. 46741

[8] Dijkstra M, van Roij R and Evans R 2000 J. Chem. Phys. 1134799

[9] Brader J M 2001 PhD Thesis University of Bristol

[10] Leal Calderon F, Bibette J and Biasis J 1993 Europhys. Lett. 23653

[11] Ilett S M, Orrock A, Poon W C K and Pusey P N 1995 Phys. Rev. E 511344

[12] Brader J M and Evans R 2000 Europhys. Lett. 49678

[13] Brader J M, Dijkstra M and Evans R 2001 Phys. Rev. E 63041405

[14] de Hoog E H A and Lekkerkerker H N W 1999 J. Phys. Chem. B 1035274

[15] de Hoog E H A, Lekkerkerker H N W, Schulz J and Findenegg G H 1999 J. Phys. Chem. B 10310657

[16] Evans R 1979 Adv. Phys. 28143

[17] Evans R 1992 Fundamentals of Inhomogeneous Fluids ed D Henderson (New York: Dekker) p 85

[18] Rosenfeld Y 1989 Phys. Rev. Lett. 63980

[19] Rosenfeld Y, Schmidt M, Löwen H and Tarazona P 1996 J. Phys.: Condens. Matter 8 L577

[20] Rosenfeld Y, Schmidt M, Löwen H and Tarazona P 1997 Phys. Rev. E 554245

[21] Tarazona P and Rosenfeld Y 1997 Phys. Rev. E 55 R4873

[22] Tarazona P 2000 Phys. Rev. Lett. 84694

[23] Cuesta J A 1996 Phys. Rev. Lett. 763742

[24] Cuesta J A and Martinez-Raton Y 1997 Phys. Rev. Lett. 783681

[25] Schmidt M 1999 J. Phys.: Condens. Matter 1110163

[26] Schmidt M 2001 Phys. Rev. E 63 010101(R)

[27] Bolhuis P and Frenkel D 1994 J. Chem. Phys. 1019869

[28] Schmidt M 2001 Phys. Rev. E 63 050201(R)

[29] Schmidt M and von Ferber C 2001 Phys. Rev. E 64051115

[30] Schmidt M and Denton A R 2002 Phys. Rev. E 65021508

[31] Schmidt M, Löwen H, Brader J M and Evans R 2000 Phys. Rev. Lett. 851934

[32] Brader J M, Evans R, Schmidt M and Löwen H 2002 J. Phys.: Condens. Matter 14 L1

[33] Groh B and Mulder B 2000 Phys. Rev. E 613811

[34] Percus J K 1976 J. Stat. Phys 15505

[35] Cuesta J A, Martinez-Raton Y and Tarzona P J. Phys.: Condens. Matter (special issue on density functional theory)

Cuesta J A, Martinez-Raton Y and Tarzona P 2002 Preprint cond-mat/0205256

[36] Brader J M and Evans R 2002 Physica A 306287

[37] A closely related, general scheme for constructing a free-energy functional for the effective one-component fluid and the corresponding hierarchy of effective direct correlation functions is given by

Cuesta J A and Martinez-Raton Y 1999 J. Phys.: Condens. Matter 1110107

[38] Roth R, Evans R and Dietrich S 2000 Phys. Rev. E 625360

[39] Roth R, Evans R and Louis A A 2001 Phys. Rev. E 64051202

[40] Rosenfeld Y 1988 J. Chem. Phys. 894272

[41] Hansen J P and McDonald I R 1986 Theory of Simple Liquids 2nd edn (London: Academic)

[42] Fisher M E and Widom B 1969 J. Chem. Phys. 503756

[43] Evans R, Henderson J R, Hoyle D C, Parry A O and Sabeur Z A 1993 Mol. Phys. 80755

[44] Evans R, Leote de Carvalho R J F, Henderson J R and Hoyle D C 1994 J. Chem. Phys. 100591

[45] Dijkstra M and Evans R 2000 J. Chem. Phys. 1121449

[46] Archer A J and Evans R 2001 Phys. Rev. E 64041501

[47] Dijkstra M, private communication Dijkstra M and van Roij R 2002 Preprint

[48] Louis A A 2001 Phil. Trans. R. Soc. A 359939 see appendix A

[49] Hall K R 1972 J. Chem. Phys. 572252

[50] Bolhuis P G, Louis A A and Hansen J-P 2002 Preprint and cond-mat/020606 